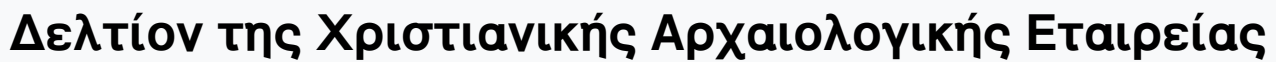

Tó 17 (1994)

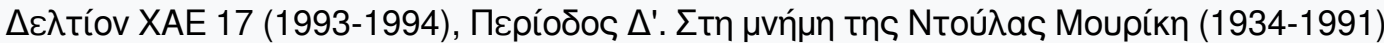

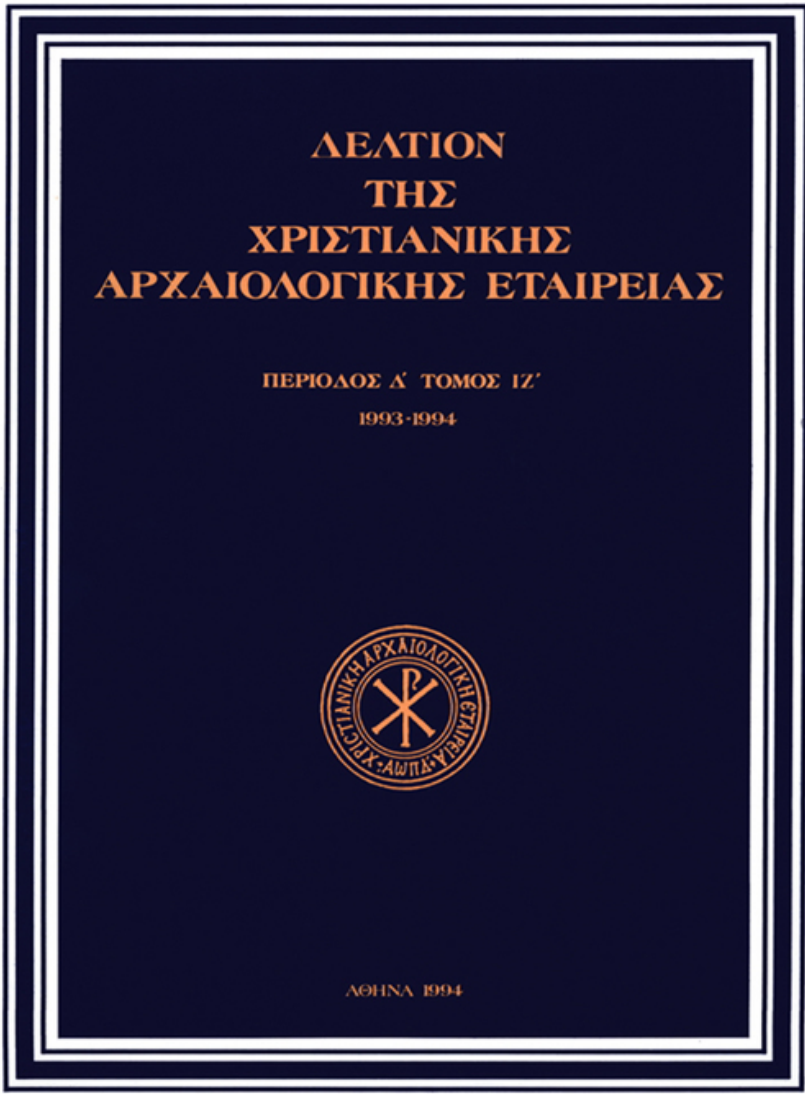

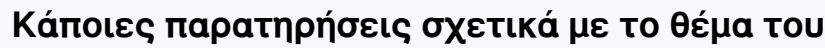

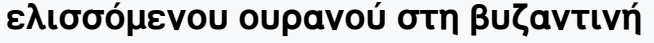

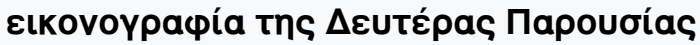

Victoria KEPETZI

doi: $10.12681 /$ dchae.1095

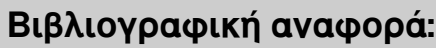

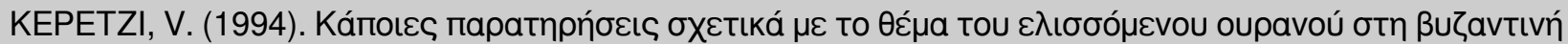

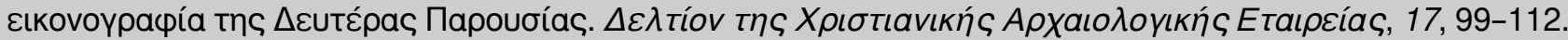
https://doi.org/10.12681/dchae.1095 


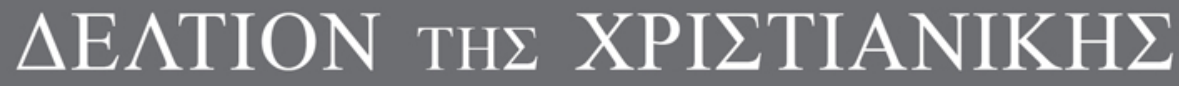 APXAIO $\Lambda$ OГIKH $\Sigma$ ETAIPEIA $\Sigma$}

Quelques remarques sur le motif de l'enroulement du ciel dans l'iconographie byzantine du Jugement

Dernier

Victoria KEPETZI

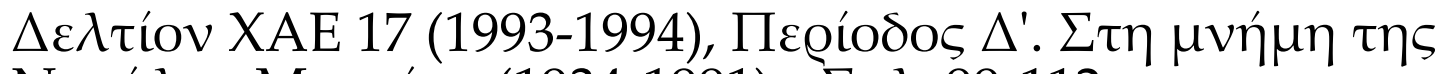

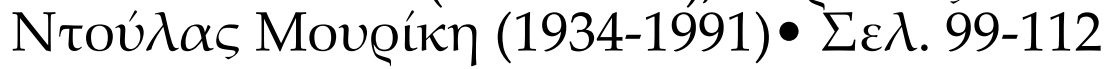

A@HNA 1994 


\section{QUELQUES REMARQUES SUR LE MOTIF DE L'ENROULEMENT DU CIEL DANS L'ICONOGRAPHIE BYZANTINE DU JUGEMENT DERNIER}

$\mathrm{L}$ La petite église voûtée à nef unique de Saint-Georges de Babylas, au Sud-Est de Néapolis, à Epidavros Liméra, située près du village de Lachi, présente un intéressant décor peint qui couvre les trois murs ainsi que la voûte du narthex. Il s'agit surtout d'une ensemble de fresques qui illustrent le cycle du Jugement Dernier et qui, en fonction de données iconographiques et stylistiques, peuvent être datées de la première moitié du XIVe siècle ${ }^{1}$. La scène qui nous occupera ici, et que $j$ 'ai choisi de présenter en hommage à la mémoire de Doula Mouri$\mathrm{ki}$ - l'un des premiers chercheurs à avoir contribué à l'étude du Jugement Dernier dans les monuments grecs $^{2}$ - sera celle des anges enroulant le ciel (Fig. 1). Cette scène est localisée sur le mur ouest, et on lit, audessus, en caractères blancs assez soignés, l'inscription: I ANГГE $\mathrm{I}$ TI $\Lambda[I \Sigma] \Sigma O N T E \Sigma$ TON O(YPANON). On sait que ce sujet iconographique est l'illustration du verset 6,14 de 1'Apocalypse. Dans un article paru en 1969, Herbert Hunger a tracé l'histoire du verset d'Isaïe 34, 4 que l'Apocalypse reprend ${ }^{3}$. A la fin de son texte, l'auteur s'interroge sur le fait que les anges qui accompagnent dans l'iconographie le ciel qui s'enroule - présents de façon quasi constante dans tous les décors byzantins du Jugement Dernier - ne sont pas mentionnés dans ce verset, non plus que dans les textes dérivés ${ }^{4}$. La présente note aura pour but d'apporter quelques précisions sur la représentation de ce sujet et de poser quelques questions sur la typologie de ces anges.

Dans l'iconographie habituelle du Jugement Dernier, et plus précisément dans celle qui reproduit la composition "monumentale $"^{5}$ du sujet, la tradition ne retient, parmi les différents phénomènes qui, dans les textes, annoncent la fin des temps ${ }^{6}$, que l'enroulement du ciel et les anges buccinateurs. Le ciel est enroulé, la plupart des fois, par un ange seul qui, figuré en mouvement et, parfois même, se retournant (Fig. 2), semble lancer dans le ciel un rouleau, le $\beta$ r $\beta \lambda$ iov des textes apocalyptiques. Ainsi, que se soit dans une composition serrée, reproduite sur les surfaces verticales comme dans l'église de la Vierge de Chalkéon à Thessalonique, dans les manuscrits (Par. gr. 74 , Hortus Deliciarum) et sur les icônes (les deux icônes du Sinaii), ou, plus libre, sur les parties hautes des églises (église ossuaire de Bačkovo et église de Sion à Aténi) - ouvres qui suivent plus ou moins les mêmes principes

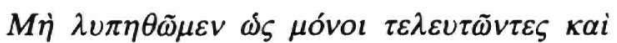

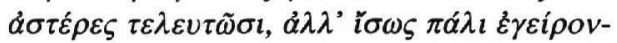

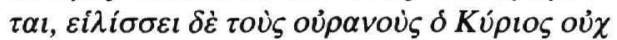

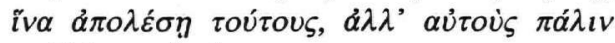

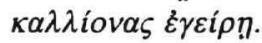

(Cyrille de Jérusalem, 15ème Catéchèse baptismale III, 4-5)

1. Le décor de cette église, présenté au XVIIIe Congrès International des études byzantines (Moscou 1991. Résumés de communications, I, p. 529) sous le titre "A propos du décor peint d'une église de Laconie", fera l'objet d'un article examinant la place de ce Jugement Dernier dans la tradition picturale. Brève description du décor dans N. B. Drandakis, N. Gkiolès, E. Dori, S. Kalopissi, V. Kepetzi, Ch. Konstantinidi, M. Konstantoudakiet M. Panagiotidi,

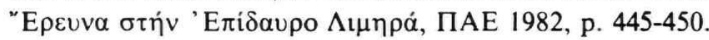

2. D. Mouriki, An Unusual Representation of the Last Judgment in a Thirteenth Century Fresco at St. George near Kouvaras in Attica, $\triangle$ XAE $\Delta^{\prime}-H^{\prime}(1975-1976)$, p. 145-171.

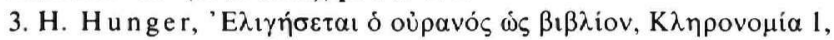
A (1969), p. 79-82. Pour la relation des notions temps et ciel, cf. H. F. We iss, Untersuchungen zur Kosmologie des hellenistischen und palästinischen Judentums, Berlin 1966, p. 18, 19

4. Hunger, op.cit., p. 81, n. 4. G. Millet, La Dalmatique du Vatican, Paris 1945, p. 15. Pour l'angélologie de cet exégète, cf. W. Cramer, Die Engelvorstellungen bei Ephraem dem Syrer, OCA 173 (1965), et p. 127 et 128 pour son caractère liturgique.

5. Pour les deux traditions dans le développement iconographique du Jugement Dernier, voir la thèse de Shige buini Tsuji, The Study of the Byzantine Gospel Illustrations in Florence, Laur. Plut. VI 23 and Paris, Bibl. Nat. cod. gr. 74, Princeton 1968, p. 53, 54 n. 42. En ce qui concerne l'illustration originelle du cycle du Jugement Dernier dans la peinture murale à Constantinople, cf. K. Weitzmann, Byzantine Miniature and Icon Painting in the Eleventh Century, The Proceedings of the XIIIth International Congress of Byzantine Studies (Oxford, 5-10 September 1966), Londres 1967, p. 207-224; id e m, Studies in Classical and Byzantine Manuscript Illumination, Chicago 1971, p. 301, 304. Pour la confrontation des deux compositions monumentales du Jugement Dernier à des scènes énonçant la fin des temps illustrées littéralement dans le Par. gr. 74, cf. S. Der Nersessian, Recherches sur les miniatures du Parisinus graecus 74, Festschrift für Otto Demus. Zum 70. Geburtstag, JÖB 21 (1972), p. 110 n. 8.

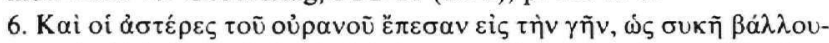

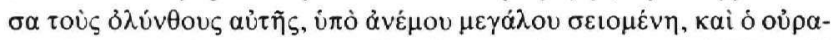

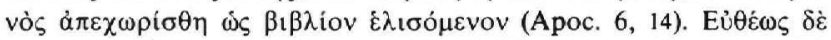

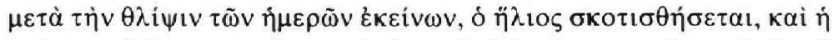

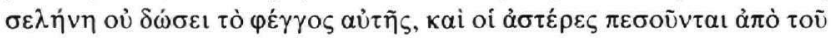

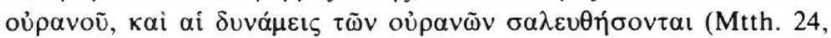
29). P. A. Underwood (The Kariye Djami, New York 1966, 1, p. 201) comprend l'enroulement du ciel comme équivalent aux signes annonçant dans Mtth. 24, 29 la Seconde Venue. Pour leur illustration, presque toujours littérale dans le texte évangélique, cf. T. Velma ns, Le tétraévangile de la Laurentienne, Paris 1971, p. 32, fig. 106 (Mtth. 24, 26-31) et où le v. 28 est illustré au fo 50r par des sarcophages: Tsuji, op.cit., p. 49. 
compositionnels pour la scène du Jugement Dernier ${ }^{7}$ sous la forme où celle-ci s'est cristallisée dès avant le XIe siècle - l'ange exécute des mouvements qui évoquent plutôt le déploiement du rouleau du ciel. Cela a, par ailleurs, entraîné l'emploi presque régulier de ce verbe "déployer" dans la plupart des descriptions de la scène, tant le mouvement esquissé est vif et la position de l'ange dans l'espace libre et dynamique ${ }^{8}$. Influencé, semble-t-il, par la figuration habituelle des rouleaux dans l'iconographie (rouleaux des prophètes), le peintre représente l'ange tenant le bout du ciel sous forme de parchemin déroulé et s'apprêtant à commencer, comme dit le texte, l'enroulement autour de l'omphalos'; l'accent étant mis sur la phase précédant l'enroulement, le texte n'est donc pas vraiment suivi à la lettre.

En peinture, la plus ancienne représentation du sujet connue à ce jour se trouve, vers 800 , dans l'église de Saint-Johann à Müstair (Fig. 4). Les deux anges qui demeurent visibles (sur les quatre qui auraient figuré à l'origine) sont presque statiques, et le rouleau du ciel, tendu et semé d'étoiles rouges et vertes, est placé à leur hauteur. Ils se tiennent debout, l'un près de l'autre, et enroulent, en le poussant, le ciel figuré en un grand

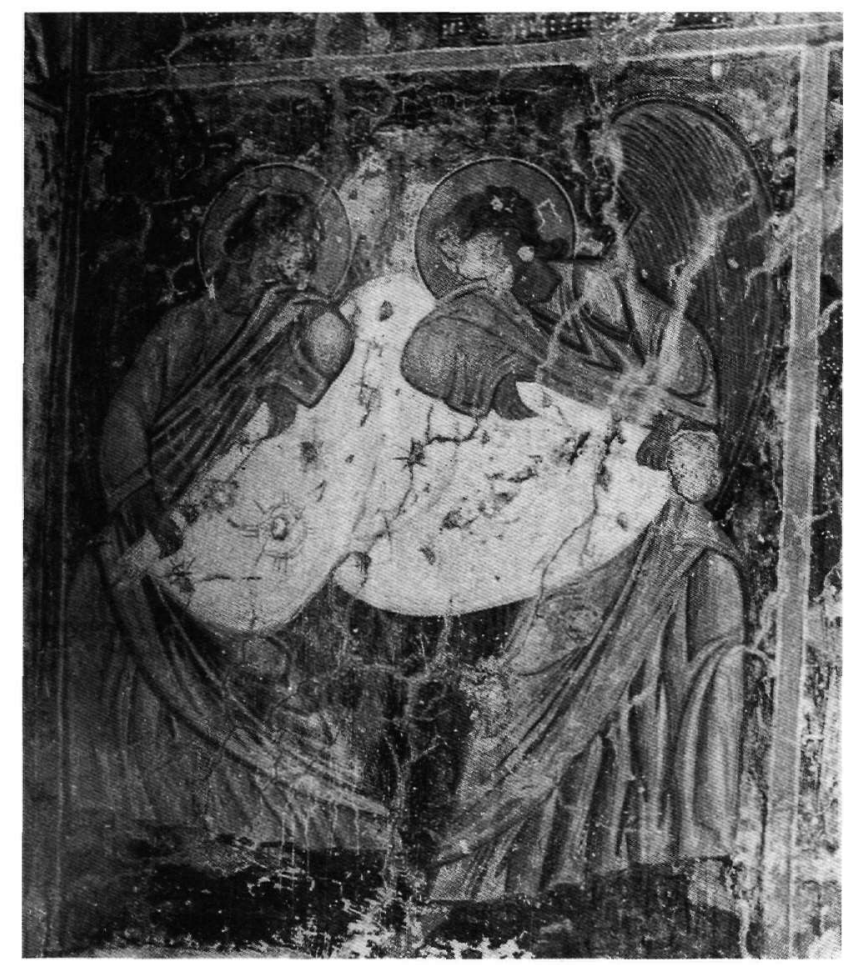

Fig. 1. Eglise de Saint-Georges de Babylas. Anges enroulant le ciel.

Fig. 2. Hortus Deliciarum, Par. gr. 74, fo 251r. Jugement Dernier (dessin).

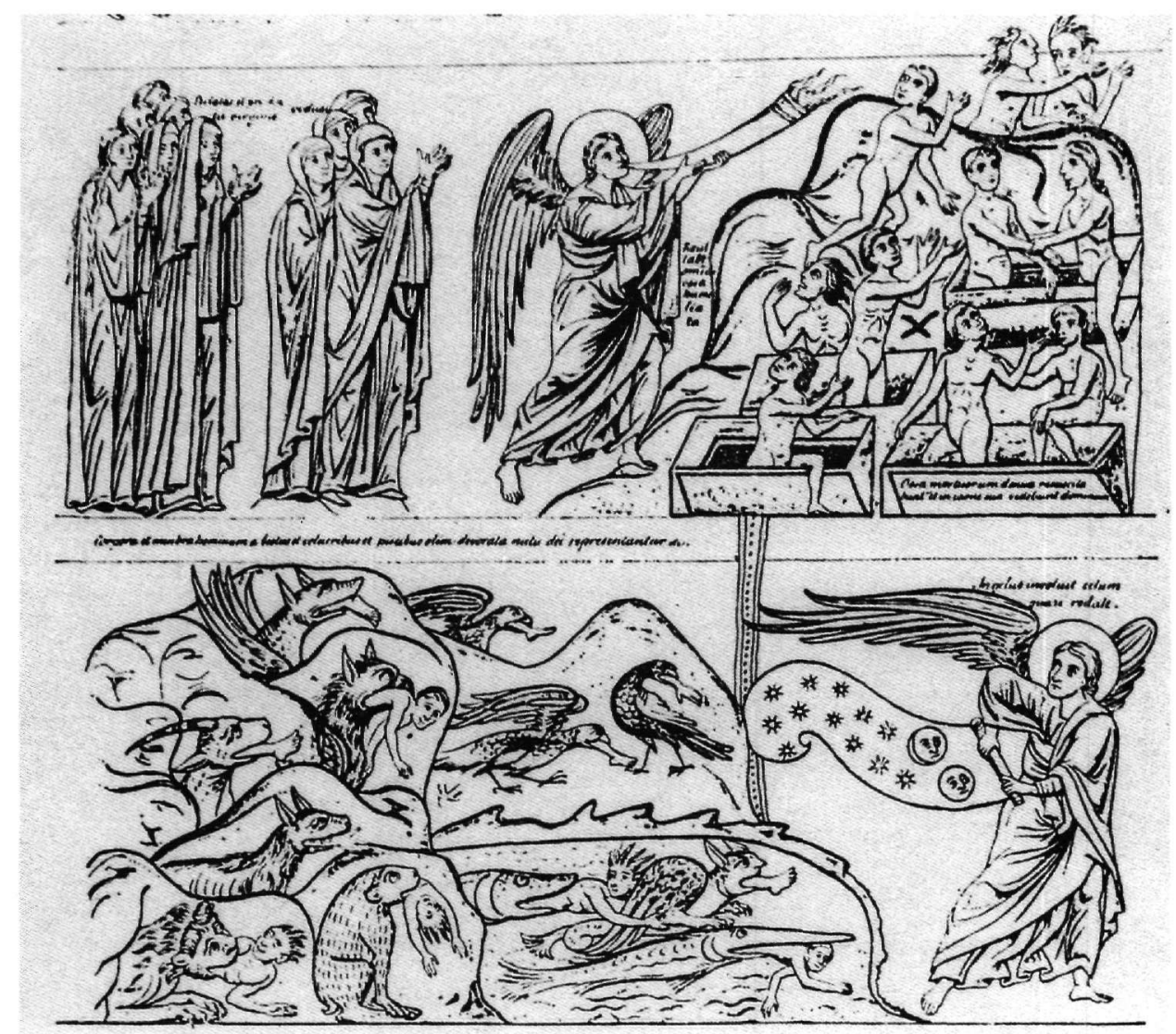


volumen à l'antique. Cette image de ce décor carolingien, étrangère à l'iconographie occidentale du Jugement Dernier, semble témoigner d'une influence byzantine directe ${ }^{10}$; quant à la variation du sujet qu'elle nous transmet, elle paraît, au contraire des exemples byzan-

7. Pour les origines du sujet (seconde moitié du VIIIe siècle), ainsi que pour une bibliographie des deux exemples, mentionnés $\mathrm{cf}$. B. B renk, Die Anfänge der byzantinischen Weltgerichtsdarstellung, BZ 57 (1964), p. 106-126; ide m, Tradition und Neuerung in der christlichen Kunst des ersten Jahrtausends, Wien 1966, p. 79-93. Pour les icônes

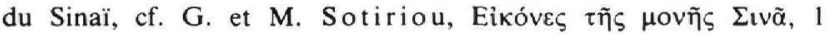
(planches), Athènes 1956, pl. 150, 151, et également Weitzman n Studies... (note 5), p. 301, 304, 306, pl. 303, 304. Pour le Hortus Deliciarum (XIIe siècle) et les rapports de son Jugement Dernier avec l'iconographie byzantine voir S. G. Tsu ji, Hortus Deliciarum et Mileseva. Esquisse d'une étude sur l'iconographie du Jugement Dernier, Orient XXI (1985), p. 62, n. 4, p. 63, fig. 2. Pour Bačkovo, cf. infra note 8. Pour l'église d'Aténi (1060-1080), où figure cette même illustration (sur la clef de la voûte de la conque ouest au dessus de la Déisis du Jugement Dernier), cf. T. Virsaladze, Rospisi Atenskogo Ciona, Tbilissi 1984, p. 14, pl. 103, sch. 10.2 que reproduit T. Velmans, L'image de la Déisis dans les églises de Géorgie et dans le reste du monde byzantin, CahArch 31 (1983), p. 150, fig. 29. Pour une datation différente du décor (1093-1096) voir G. A b r a michvili, La datation

Fig. 3. Icône du Sinaï. Jugement Dernier.

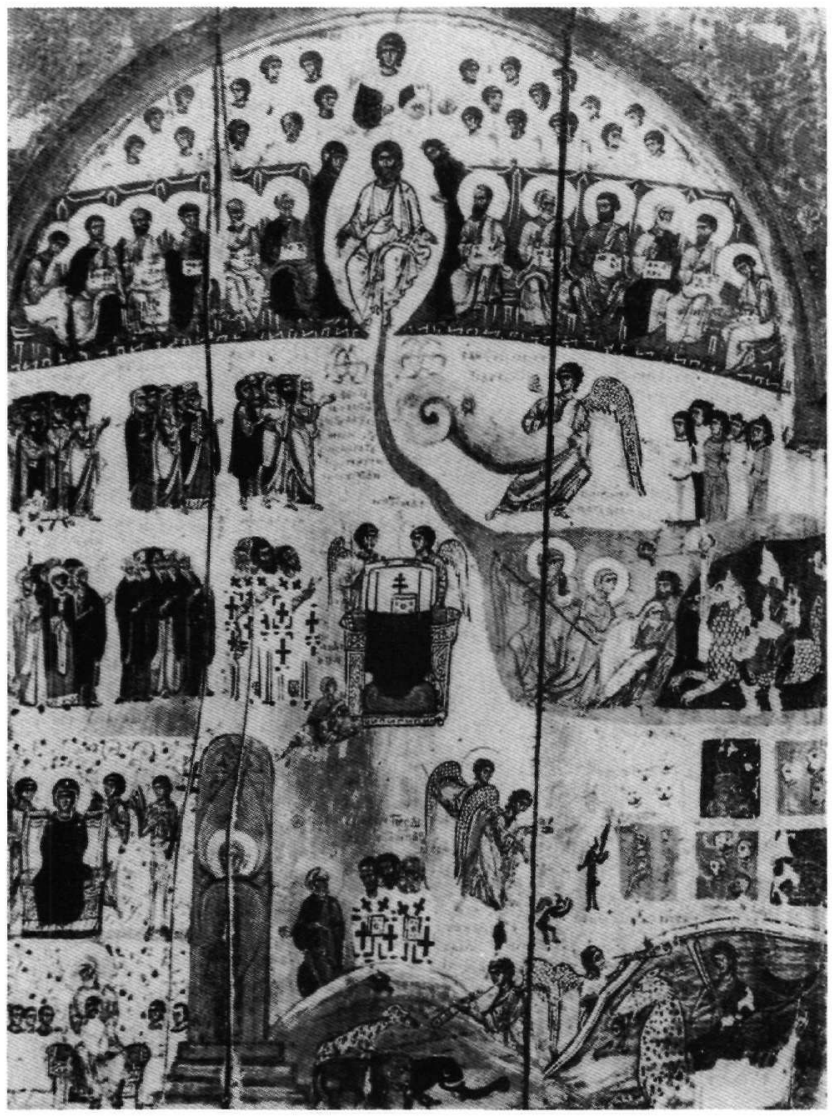

des fresques de la cathédrale d'Aténi, Zograf 14 (1983), p. 21. 8. Un des exemples les plus évocateurs (suggéré aussi par son emplacement sur la voûte), est le long rouleau au-dessus de l'ange dans le narthex de l'église ossuaire de Bačkovo (3e quart du XIIe s.): E. Baka lova, Bačkovskata Kostnica, Sofia 1977, p. 242, fig. 152. Pour ce décor du peintre de culture géorgienne Jean Iviropoulos, et considéré habituellement du XIIe siècle, il a été proposée également une datation vers la fin du XIe siècle: D. Mouriki, The Formative Role of Byzantine Art on the Artistic Style of the Cultural Neighbors of Byzantium, XVI. Internationaler Byzantinistenkongress, Akten I/2, JÖB 31/2 (1981), p. 733, n. 21. De son côté, L. Mavrodinova penche pour le 2e quart du XIIe siécle: Sur la datation des peintures murales

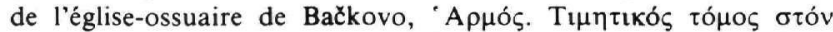

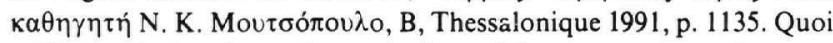
qu'il en soit, la scène qui nous intéresse ici occupe, comme dans nos exemples géorgiens (XIe, XIIe s.), la voûte au-dessus des apôtres (note 20 infra) - encore que ce choix puisse s'expliquer par la contrainte architecturale de l'espace à décorer. On retrouve la même disposition pour l'ange sur la voûte représentant le ciel, dans des églises plus tardives (cf. notes 22 et 44 ).

9. Hunger, op.cit., p. 79, n. 2. Pour un aperçu de cette forme de manuscrit qui figure ici le ciel, cf. note suivante.

10. Ainsi que la Déisis de même décor, Brenk, Tradition und Neuerung (note 7), p. 111, fig. 7, 9, pl. 33; L. Birchler, Zur karolingischen Architektur und Malerei in Münster-Müstair, Akten zur III. Internationalen Kongress für Frühmittelalterforschung, Olten-Lau-

Fig. 4. Eglise de Saint-Johann, Müstair. Anges enroulant le firmament.

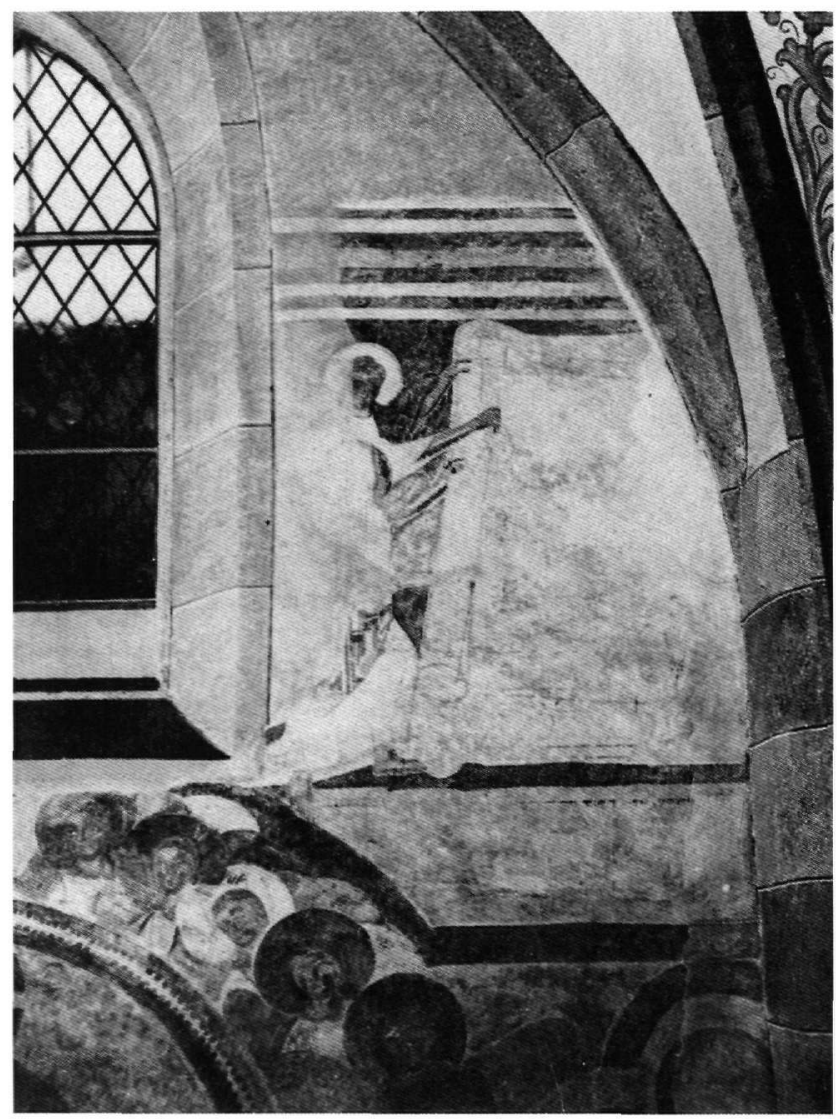




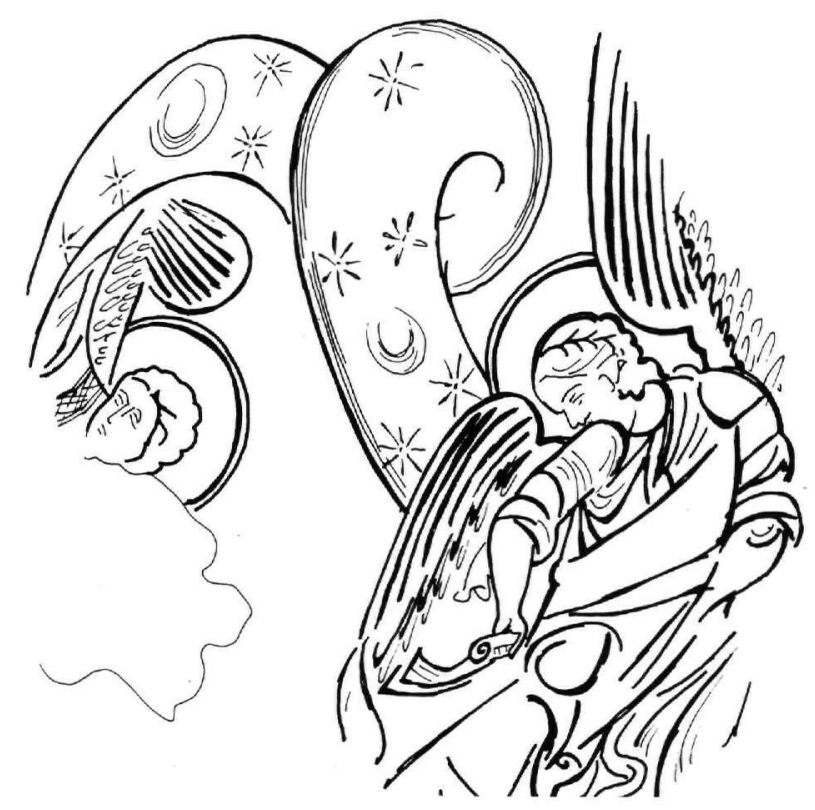

Fig. 5. Eglise de la Mère de Dieu, Axtala (Géorgie). Anges enroulant de ciel (croquis).

tins plus tardifs du XIe siècle que l'on vient de voir, suivre très fidèlement le texte de l'Apocalypse évoqué. Les premiers exemples qui suivent chronologiquement Müstair, ceux de la tradition médio-byzantine, n'ont pas retenu ce schéma iconographique de deux anges à rouleau tendu, lui préférant celui de l'ange seul, en mouvement (Fig. 2-3). Ainsi, dans les diverses figurations de ce mềme sujet qui a tant séduit les peintres ${ }^{11}$, le caractère "dynamique» de la démarche de l'ange - une de ses constantes iconographiques - est souvent frappant. Parfois même, comme dans l'église de la Vierge d'Axtala en Géorgie (début XIIIe s.) (Fig. 5), on peut aboutir à des anges qui se détournent tout en avançant en grandes enjambées - ce que soulignent les plis mouvementés de leurs vêtements - entraînant derrière eux le firmament $^{12}$.

Si on ne connaît pas d'analogies contemporaines de cette composition de Müstair, certains éléments constitutifs de celle-ci se retrouvent cependant, bien plus tard, et cela non seulement dans des scènes du Jugement Dernier. On en rencontre ainsi dans les Homélies de Jacques de Kokkinobaphos qu'illustrent le Vat. gr. 1128 et le Par. gr. 1208, manuscrits constantinopolitains du 2ème quart du XIIe siècle. Dans une représentation majestueuse de ce dernier manuscrit (Fig. 6), le ciel visible ${ }^{13}$ - que rend une longue bande tendue à surface constellée où figurent la lune à gauche, le soleil à droite - est soulevé, comme un rideau, par deux anges se tenant debout aux deux extrémités et encadrant d'autres anges,

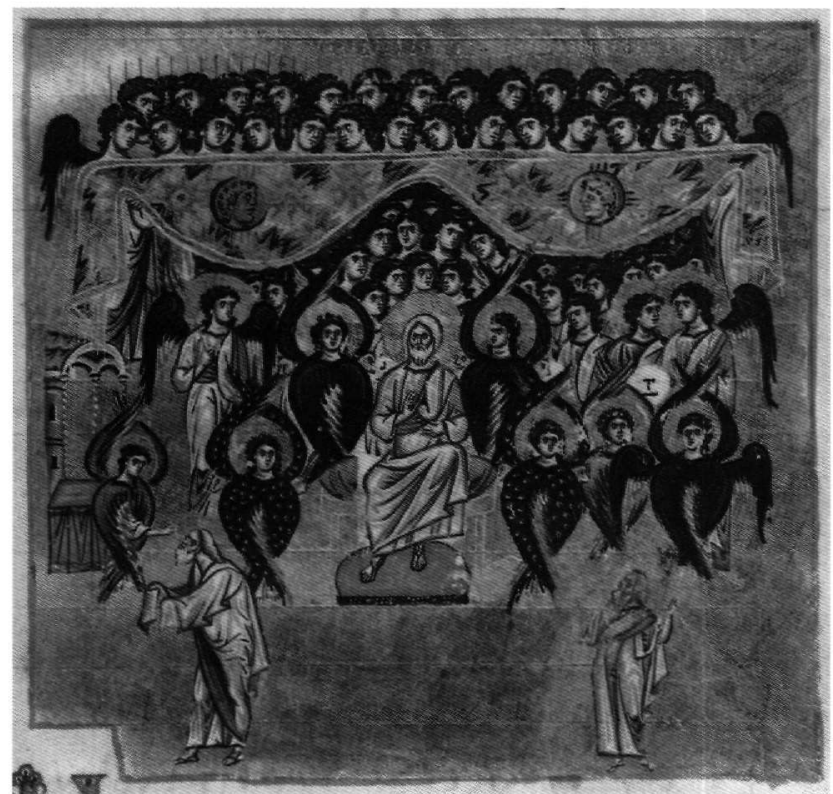

Fig. 6. Par. gr. 1208, $f^{\circ} 162$ r. Vision d'Isaïe (photo Bibliothèque Nationale de Paris).

ciel que Grabar a rapproché de la vision "optique» de l'iconographie du firmament ${ }^{14}$ qu'il oppose à sa représentation "scientifique». Cette dernière nous est, par exemple, transmise par l'image de la bande céleste se-

sanne 1954, p. 229. Les influences byzantiries sont également attestées dans des décors plus tardifs en Occident, lorsque figure ce même sujet de l'ange au rouleau du ciel, comme dans la chapelle de Saint-Sylvestre auprès des Quatre Saints Couronnés à Rome (1246). G. Ma tth i a e, Pittura romana del Medioevo, Roma 1988, II, p. 139, 140, 142, fig. 133, 134. Pour le rouleau comme support matériel des textes, cf. L. W. Daly, Rotuli: Liturgical Rolls and Formal Documents, GRBS 14, 3 (1973), p. 333-338, spécialement 336 notes 15,17 et 18 .

11. Les anges enroulant le ciel sont parfois représentés à une échelle plus grande que celle du reste des figures du Jugement Dernier. Ainsi, plus grand, provenant peut-être d'un autre prototype, est l'ange d'une des icônes du Sinaï (We it z m a n n, op.cit. (note 5), fig. 304) alors que dans l'église le Vardzia en Géorgie (1184-1185), ils sont même démesurément grands. T. Velmans, La koine grecque et les régions périphériques orientales du monde byzantin, XVI. Internationaler Byzantinistenkongress, Akten I/2, JÖB 31/2 (1981), p. 718, n. 190. Ce sujet peut avoir des variations formelles, voire disparaître. On note déjà l'absence du motif sur une composition peinte du Jugement Dernier du Xe siècle (cf. note 41) puis sur l'ivoire du Victoria et Albert Museum (Xe ou XIe s.): Brenk, Tradition und Neuerung (note 7), p. 84, n. 7, pl. 23; We it z m a n n, Byzantine Miniature and Icon Painting, op.cit. (note 5), p. 304, n. 72, ainsi que sur les décors plus tardifs, comme dans certaines églises du Magne, entre autres à Episcopie $(\mathrm{N}$.

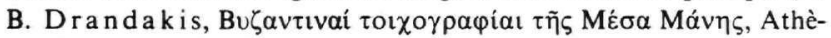
nes 1964, p. 83, fig. 5), datable, non plus du XIIe, mais du ler quart du

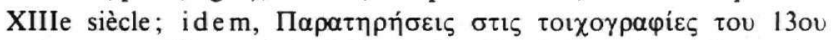

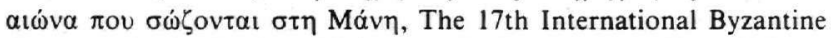
Congress, Major Papers, Washington D.C., 1986, New York 1986, p. 


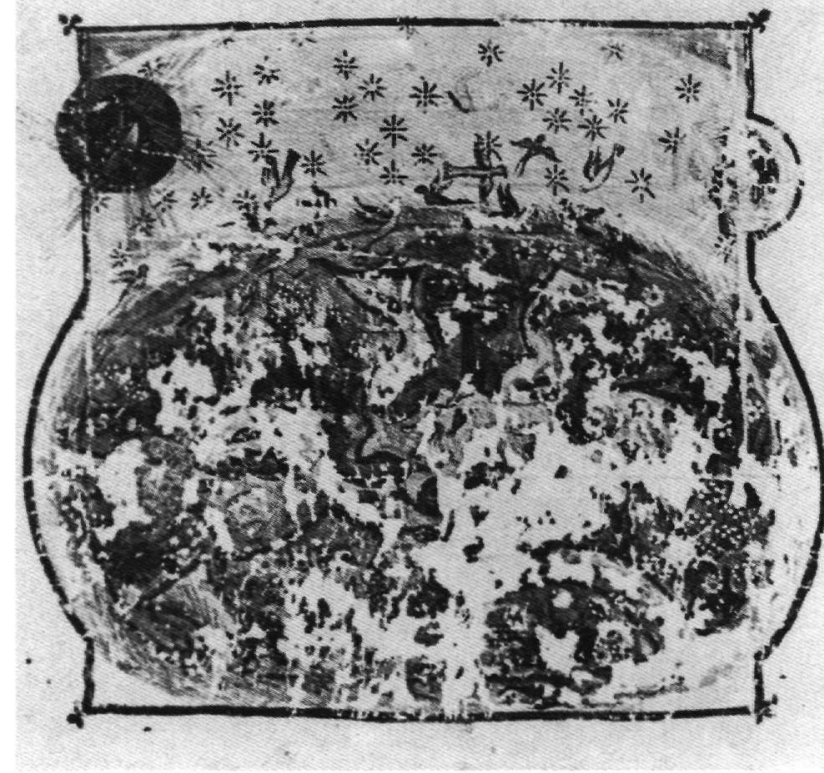

Fig. 7. Vat. gr. 747, fo 17r. La Création de l'Univers.

Fig. 8. Eglise de Saint-Nicolas tis Stégis, Chypre. Les anges enroulant le ciel.
692) ou en Géorgie (Timothesubani, XIIIe siècle), Vel m a n s, op.cit., L'image de la Déisis (note 7), p. 149.

12. N. Thierry, Le Jugement Dernier d'Axtala, Bedi Kartlisa 40 (1980), p. 156, fig. 3, 4, insiste sur la part d'innovation dont fait montre ici cet atelier (p. 168), Cf. V. Lidov, The Mural Paintings of Akhtala, Moscou 1991, p. 21, 59, sch. 5 fig. 41 et p. 105-109 pour un essai d'évaluation de ce décor par rapport à la tradition byzantine et une datation plus précise (1205-1216). Mentionnons, à propos de l'origine constantinopolitaine des disques qui figurent sur le rouleau du ciel, l'article de E. C. Swart z, The Whirling Disc, Zograf 8 (1977), p. 24 , fig. 1, et p. 26 n. 13, p. 27 n. 23 . Je remercie chaleureusement Mlle I. Lambropoulou à qui je dois le dessin de la Fig. 5 .

13. Pris dans l'illustration de la vision d'Isaïe: Vat. gr. 1162 ( $\left.f^{\circ} 119 \mathrm{v}\right)$ C. St or na jolo, Miniature delle Omilie di Giacomo Monaco, Roma 1910, pl. 52. De ces deux manuscrits identiques, le plus ancien est censé être celui de Paris (env. 1130), I. Hutter, Die Homelien des Mönches Jakobos und ihre Illustrationen, Vat. grec. 1162-Par. grec. 1208, Wien 1970. Je remercie chaleureusement Mme Irgmard Hutter et Mlle Suzy Dufrenne pour avoir eu la gentillesse de me communiquer des photocopies de cette thèse non publiée, et des informations sur le manuscrit de Paris. Pour la datation et l'appréciation du milieu auquel étaient adressées ses Sermons illustrées ainsi que son implication sur leur décor, voir J. C. A nde r s on, The Illustrated Sermons of James the Monk: their Dates, Order and Place in the History of Byzantine Art, Viator 22 (1991), p. 85 et 95-101. Je remercie le R. P. Christopher Walter pour m'avoir indiqué cet article.

14. Cf. A. Grabar, L'iconographie du Ciel dans l'art chrétien de l'Antiquité et du haut moyen âge, CahArch 30 (1982), p. 10-12 et fig. 9. Analyse iconographique de la composition de Kokkinobaphos dans la thèse de Hutter, op.cit., p. 342-357.

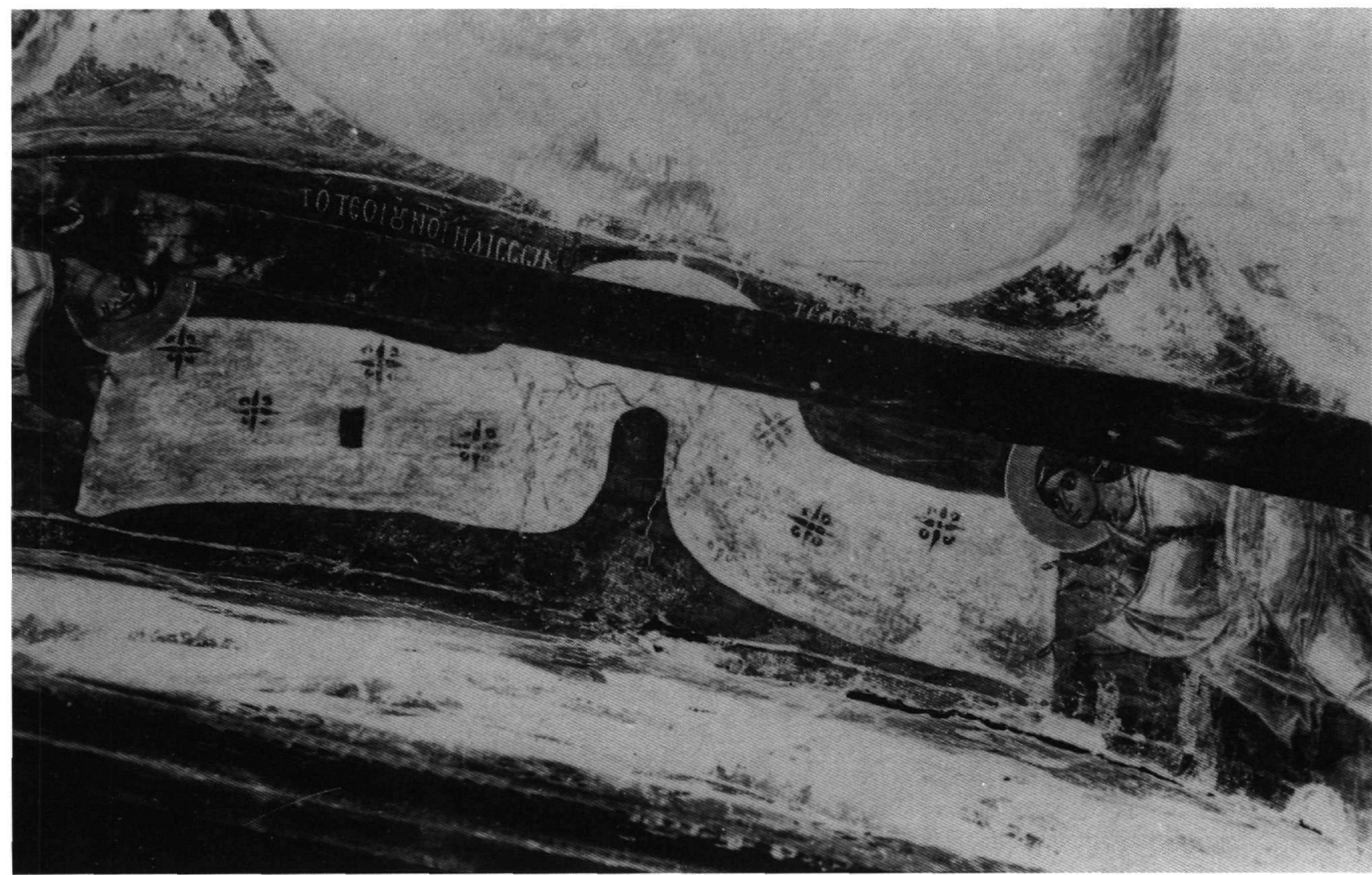


mée d'étoiles, portant symétriquement les effigies du soleil et de la lune, et épousant la courbe de la sphère terrestre dans l'Octateuque du Vat. gr. 747 du XIe siècle (Fig. 7) qu'on avait considéré comme fidèle aux prototypes de la tradition hellénistique ${ }^{15}$.

Presque contemporaine de l'illustration du plus ancien des manuscrits de Kokkinobaphos, mais d'un aspect plus archaïque, l'image des anges enroulant le ciel, dans le narthex de l'église de Saint-Nicolas tis Stégis à Kakopetria de Chypre (que l'on a cru pouvoir dater - en fonction de critères stylistiques - du début du XIIe siècle), présente des analogies avec la composition du Par. gr. 1208: la bande céleste est presque tendue avec seule une courbe au milieu - ne comporte que des étoiles rouges - à l'instar de celui de Müstair, quoique assez différentes de forme - et les anges ne font qu'en tenir les extrémités, prêts à l'enrouler selon l'inscription TOTE OI OY(PA)NOI HAICC $\Omega$ N/TE $\Omega$ C BI..ION ${ }^{16}$ (Fig. 8). Cette image occupe l'intrados de l'arc soutenant la coupole.

On ne peut, pour le moment, établir une relation entre les éventuelles influences et interférences de ces deux schémas iconographiques, celui de la miniature de Paris, création constantinopolitaine dont les origines iconographiques ne doivent pas remonter au delà du XIe siècle, et celui de la fresque de Müstair où les anges, debout, figureraient de chaque côté enroulant le firmament ${ }^{17}$. Enfin, apparentée à ces anges presque statiques semble également la composition chypriote créée à une période de contacts étroits entre l'île de Chypre et Constantinople. On se trouve avec Saint-Nicolas tis Stégis, un siècle après la première apparition connue du motif en peinture byzantine (Fig. 2-3), devant une composition qui ne décore plus des surfaces verticales, pour lesquelles elle aurait été inventée (on rencontre cependant cette composition sur une surface incurvée: Aténi, Bačkovo), mais voûtées. La raison principale du choix de deux anges pour cette scène doit-elle être cherchée dans la volonté du peintre de l'harmoniser avec les surfaces fortement incurvées du décor (tel l'intrados d'un arc ou d'une fenêtre), objectif qui semble déjà avoir guidé, au XIe siècle, le peintre d'Aténi (même s'il ne s'agit encore que d'un ange unique), le peintre chypriote jouant admirablement avec la flexibilité de la forme plastique du rouleau du ciel? Quoi qu'il en soit, la composition de deux anges dans une scène du Jugement Dernier - plus harmonieuse, bien que moins réaliste que celle de l'ange unique ${ }^{18}$ - connaîtra, surtout à l'époque paléologue, une grande diffusion, souvent sur des surfaces fortement incurvées, autant en peinture murale que dans les manuscrits lorsque leurs scènes s'inspirent directement des décors monumentaux.
Des exemples de deux anges enroulant le ciel se retrouvent également, outre à Chypre et dans les Balkans (comme on le verra plus loin), dans la périphérie byzantine. On en rencontre ainsi dans l'église de la Mère de Dieu à Axtala en Géorgie (Fig. 5) qui serait un des premiers exemples, en peinture murale, où les deux anges, habilement adaptés à la surface incurvée de l'intrados des fenêtres - emplacement peu habituel pour la figuration de scènes - se détournent pour enrouler à deux le rouleau du ciel. On distingue, entre les étoiles, deux disques polis qui, chargés d'un symbolisme plus ou moins précis, se retrouvent sur les icônes et des fresques de l'école constantinopolitaine dès l'époque comnène ${ }^{19}$.

15. Selon la thèse de K. We it zman n (The Joshua Roll. A Work of the Macedonian Renaissance, Princeton 1948, p. 31-33, 38). Pour un aperçu critique, cf. D. T selos, The Joshua Roll: Original or Copy? ArtB 32 (1950), p. 278, 290 Pour la tradition dite scientifique à laquelle cette image appartient, cf. Grabar, op.cit., p. 12. Dans ce manuscrit, le firmament, bleu ciel, semé d'étoiles dorées, se profile sur fond or ( $\left.f^{\circ} 16 v, 17 r\right)$. Pour les images, descriptions et reproductions en couleur d'un autre Octateuque proche, cf. J. Las s us, La création du Monde dans les Octateuques byzantins du douzième siècle, MonPiot 62 (1979), p. 109, 111 et 112 , pl. II.E, et p. 148 pour des indices concernant une datation éventuellement plus tardive du Vat. gr. 747. Voir également J. Lowden, The Octateuchs. A Study in Byzantine Manuscript Illustration, Pennsylvania 1992, p. 14 n. 16 et p. $61,83,89$, 91,106 pour les plus récentes tentatives de datation de ce manuscript (vers 1075) et pour une approche critique de ses liens avec son modèle iconographique, et $\mathrm{O}$. Kreste n, Oktateuch-Probleme: Bemerkungen zu einer Neuerscheinung, BZ 84-85 (1991-1992), p. 502, 503, 505, 506. Notons par ailleurs que dans l'Homélie 56 sur Matthieu, Jean Chrysostome (PG 58, 554, $\delta$ ), évoquant le Christ de la Transfiguration,

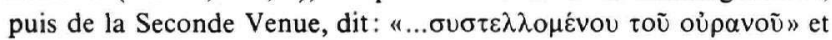

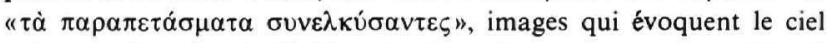
qu'on soulève dans le Kokkinobaphos: Hunger, op.cit., p. 81 ; cf. note 39 .

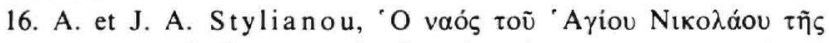
$\Sigma \tau \varepsilon \dot{\gamma} \eta \varsigma \pi \alpha \rho \alpha \dot{~ \tau ท ́ v ~ K \alpha \kappa o \pi \varepsilon \tau \rho ı ́ \alpha, ~ K u \pi \rho \Sigma \pi o v \delta ~} 10$ (1946), p. 121, fig. 2. On doit, bien sûr, lire un $\mathrm{B}$ au lieu d'un $\mathrm{X}$ dans la seconde partie de l'inscription, puisque le mot incomplet ne peut être que le "BIB $\triangle I O N$ " de l'Apoc. 6, 14. Cf. également A. et J. Stylia nou, The Painted Churches of Cyprus, London 1985, p. 59, 61.

17. Hutter, op.cit., p. 357, et non pas avant (Grabar, op.cit. (note 14), p. $11 \mathrm{n} .8$ ). La présence éventuelle de quatre anges du décor carolingien, à côté du rouleau, offrirait plus d'analogies formelles avec la composition originelle de la scène de Kokkinobaphos.

18. Il est plus probant qu'un seul ange enroule le rouleau; lorsqu'ils opèrent chacun de leur côté, le rouleau ne pourra évidemment jamais être enroulé. Ici, nos exemples se différencient donc de scènes de même inspiration, telles celles d'évangélistes, par exemple, qui, pour lire leur volumen, le déroulent de la main droite pour enrouler de la main gauche.

19. L. Hadermann Misguich, Aspects de l'ambiguïté spatiale 


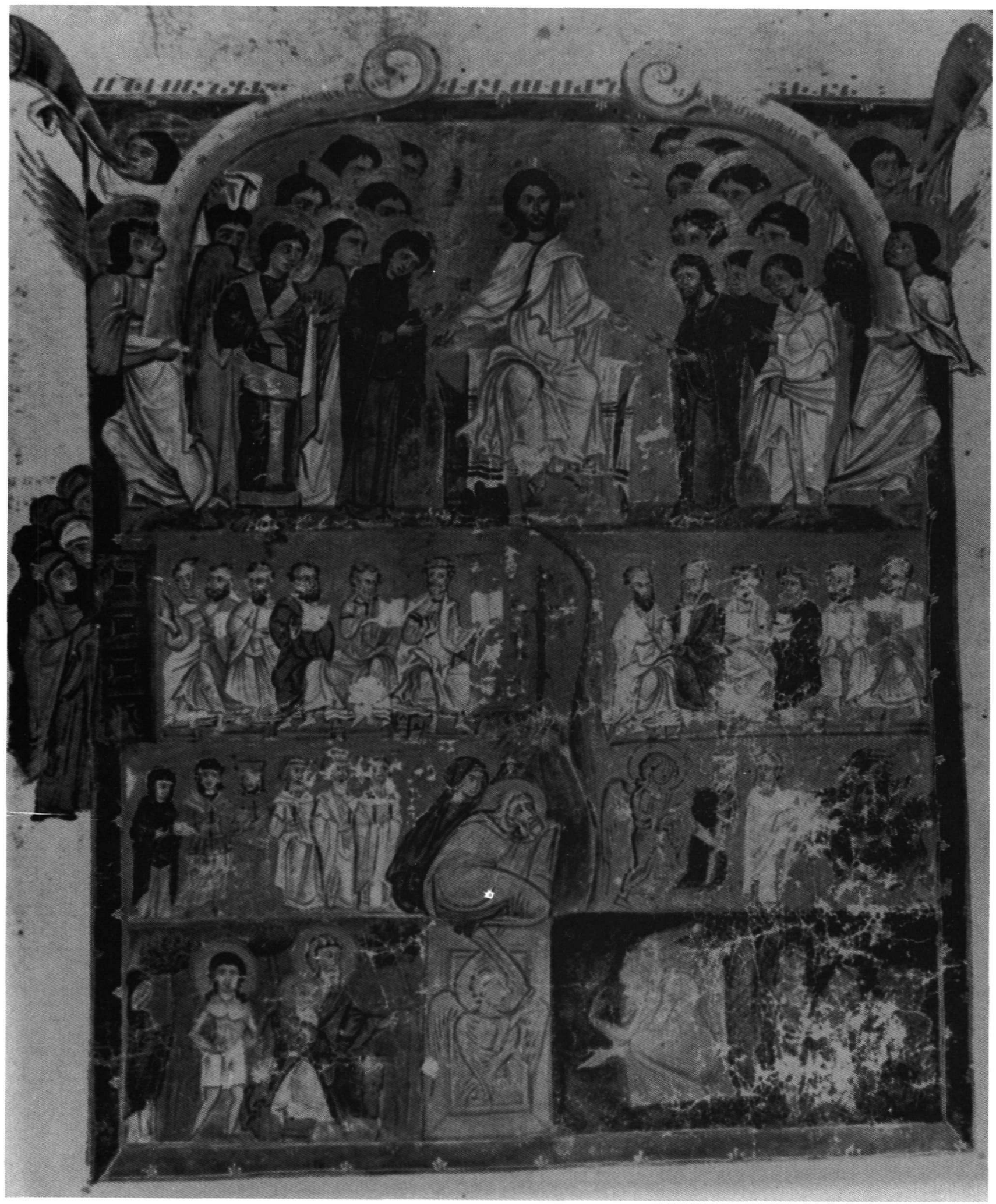

Fig. 9. Tétraévangile W 539 de la Walters Art Gallery, Baltimore, fo 109r. Jugement Dernier. 


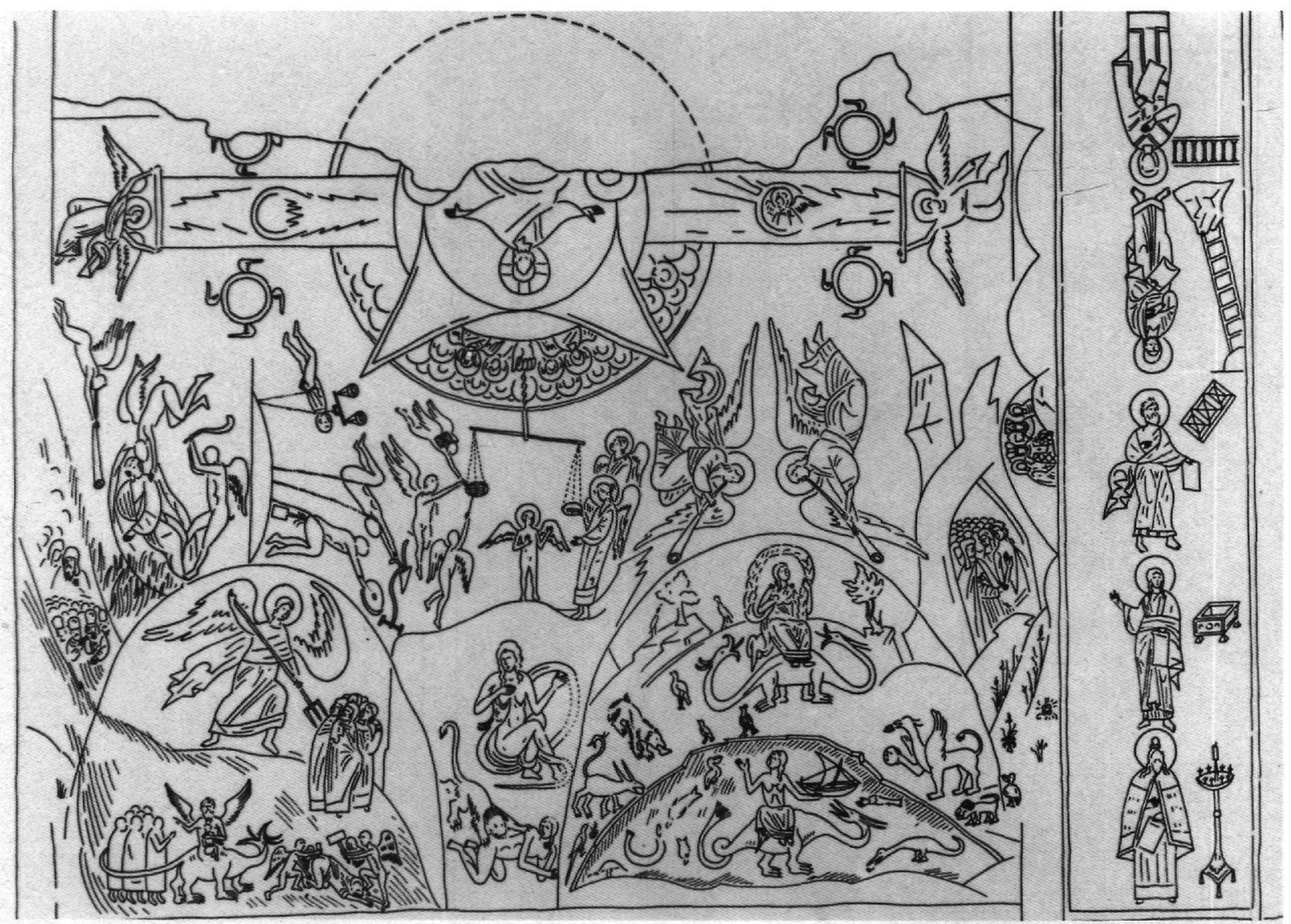

Fig. 10. Bogorodiča Ljeviška (exonarthex). Jugement Dernier (dessin).

Il semble que, par la suite, la présence de deux anges sera généralement dictée par cette volonté d'inscrire plus harmonieusement l'image dans l'espace architectural à décorer, mettant ainsi encore plus en relief leur fonction dans cette scène.

Cette nouvelle composition passe ensuite de la peinture monumentale dans les manuscrits, comme le tétraévangile de la Walters Art Gallery (W 539) de 1262 (Fig. 9). Sur cette miniature (qui s'est peut-être inspirée de schémas antérieurs) deux anges, placés dans la partie haute de la composition, comme à Aténi, debout et courbés vers le centre, tenant chacun son rouleau du ciel, sont représentés - de façon qu'on a jugé éloignée des normes byzantines $^{20}$ - au-dessus de la Déisis du Jugement Dernier et couronnent la composition. Ici, le peintre est fidèle à la logique d'utilisation pratique de cette forme de manuscrit, même s'il n'est pas impossible que l'éventuel modèle iconographique de cette variation ait pu servir également à la création du schéma où un même rouleau est tenu à chaque extrémité par deux anges.
La représentation d'un seul rouleau tenu par deux anges, comme on vient de la rencontrer à propos d'Axtala, connaîtra un succès certain dans la peinture tardobyzantine, comme en témoigne l'extension de cette nouvelle variante qui, interprétée avec plus ou moins de bonheur, se retrouve aussi bien dans les Balkans ${ }^{21}$ qu'à Chypre. Dans l'église de Bogorodiča Ljeviška (13071313) (Fig. 10), au sommet de la voûte de l'exonarthex, les extrémités du rouleau du ciel, long et tendu, sont enroulées de part et d'autre par des anges en plein vol. L'intérêt de cette composition ne réside pas seulement dans sa complexité formelle, mais aussi dans la façon ingénieuse de superposer, sur ce rouleau, la gloire sur laquelle se profile le Christ Juge, signifiant peut-être ainsi l'antériorité de l'enroulement du ciel, signe précurseur de la Seconde Parousie. Cette part d'invention compositionnelle (mais peut-être aussi sémantique) est l'oeuvre de l'atelier d'Astrapas, un peintre qui, de toute évidence, connaît la grande tradition de son temps, qu'il adapte de façon personnelle ${ }^{22}$, et qui a destiné, à cette 


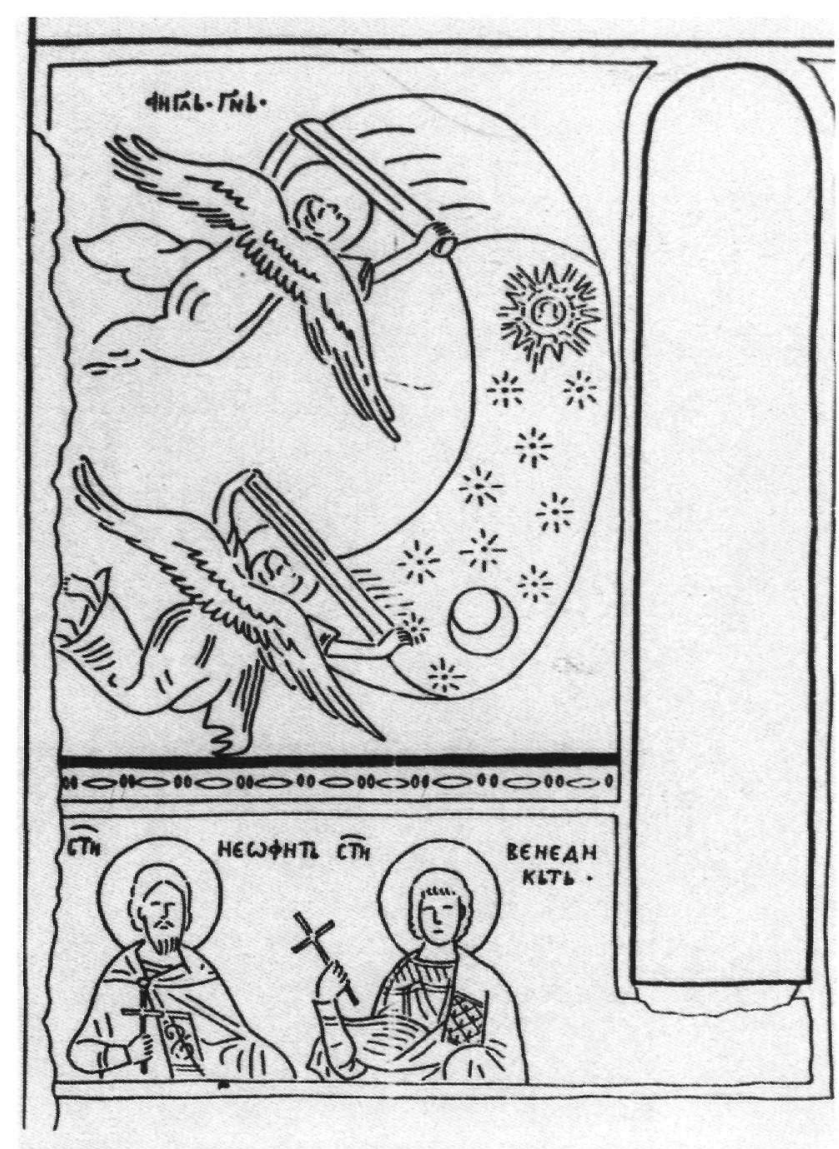

Fig. 11. Gračanica. Anges enroulant le ciel (dessin).

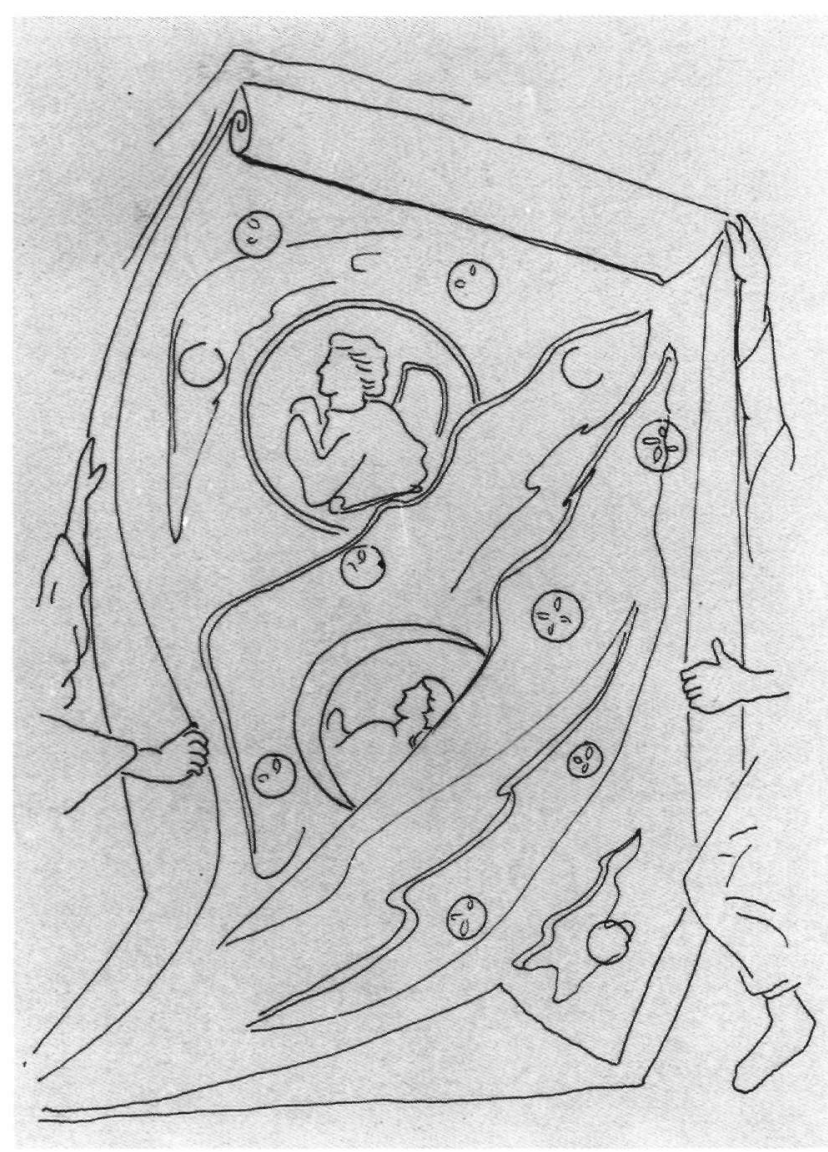

Fig. 12. Dečani. Anges enroulant le ciel (dessin). dans la peinture monumentale byzantine, XVIIIe Congrès International des études byzantines, Moscou 1991, Résumés des communications, I, p. 422-423; pour les disques cf. note 12 ainsi que Mouriki (note 8), p. 734 pour une disposition analogue des figures à l'intérieur de fenêtres dans l'église de Bačkovo qui rappelle en cela Aténi.

20. S. Der Nersessian, Miniatures ciliciennes, L'Oeil, n. 179 (novembre 1969), Études byzantines et arméniennes, Louvain 1973, I, p. 512, fig. 247; sur l'image, les rouleaux portent le v. Is. 34.4. I de m, Armenian Manuscripts in the Walters Art Gallery, Baltimore 1973, p. 19, fig. 80. Pour une plus ample analyse des éléments qui s'éloignent de la tradition byzantine, cf. K. Weit zmann, Icon Painting in the Crusader Kingdom, DOP 20 (1966), p. 59 fig. 15, qui attribue cette disposition à une influence occidentale. Pour les relations entre la peinture cilicienne et certaines icônes du Sinaï, voir également l'article de V. Pace, Armenian Cilicia, Cyprus, Italy and Sinaï Icons. Problems of Models in Medieval Armenian Culture (Armenian Texts and Studies 6), Pennsylvania 1984, p. 292 n. 15. Je ne connais pas la composition du fo 80 de l'évangile de Džruči II (Tbilissi, cod. H 1667); dans ce manuscrit géorgien du XIIe siècle, qui suit en grande partie la tradition byzantine, figureraient - couronnant la partie haute au-dessus de la Déisis de la scène du Jugement Dernier, emplacement déjà choisi pour la scène à Aténi (cf. note 7) - deux anges enroulant le ciel. Velmans, L'image de la Déisis, op.cit. (note 7), p. 151. Sur le plan formel, on aurait ici le même motif rencontré dans la peinture monumentale au début du XIIe siècle à Chypre (Fig. 7), imité peut-être de surfaces incurvées d'une église sur lesquelles se reflète mieux son caractère décoratif. Peut-être faudrait-il ainsi nuancer l'assertation de K. Weitzmann concernant les origines de ce schéma qui pourrait bien n'avoir d'occidental que l'emplacement des anges buccinateurs. Notons que des anges sonnant de la trompette figurent également sur la partie haute de ce même évangile géorgien du XIIe siècle, au fo $64 r$, liés ici à la Seconde Venue. Cf. Velmans, L'image de la Déisis, op.cit. (note 7), fig. 30. Ce même sujet, doublé de celui de l'ange avec rouleau du ciel, est disposé de façon analogue à celui de la miniature arménienne et figure à Rome, au-dessus de la Déisis du Jugement Dernier, dans un décor du XIIIe siècle à influences byzantines; cf. note 10 .

21. Pour les survivances de ce schéma dans les monuments balkaniques post-byzantins (Cathédrale Uspenski à Valdimir), cf. Tsuji, Hortus Deliciarum et Mileseva, op.cit. (note 7), p. 73 n. 6.

22. D r. Pa n ić et G. B a bić, Bogorodiča Ljeviška, Beograd 1975, p. 109,113 , dessin 30 (p. 138); la différence formelle avec la fresque presque contemporaine de Kariye Djami a été déjà notée: $\mathrm{S}$. Der

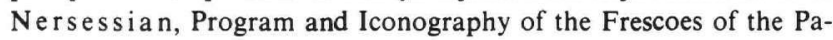
recclesion, The Kariye Djami, 4, Studies in the Art of the Kariye Djami and its Intellectual Background (ed. P. A. Underwood), Princeton 1975 , p. 326. 


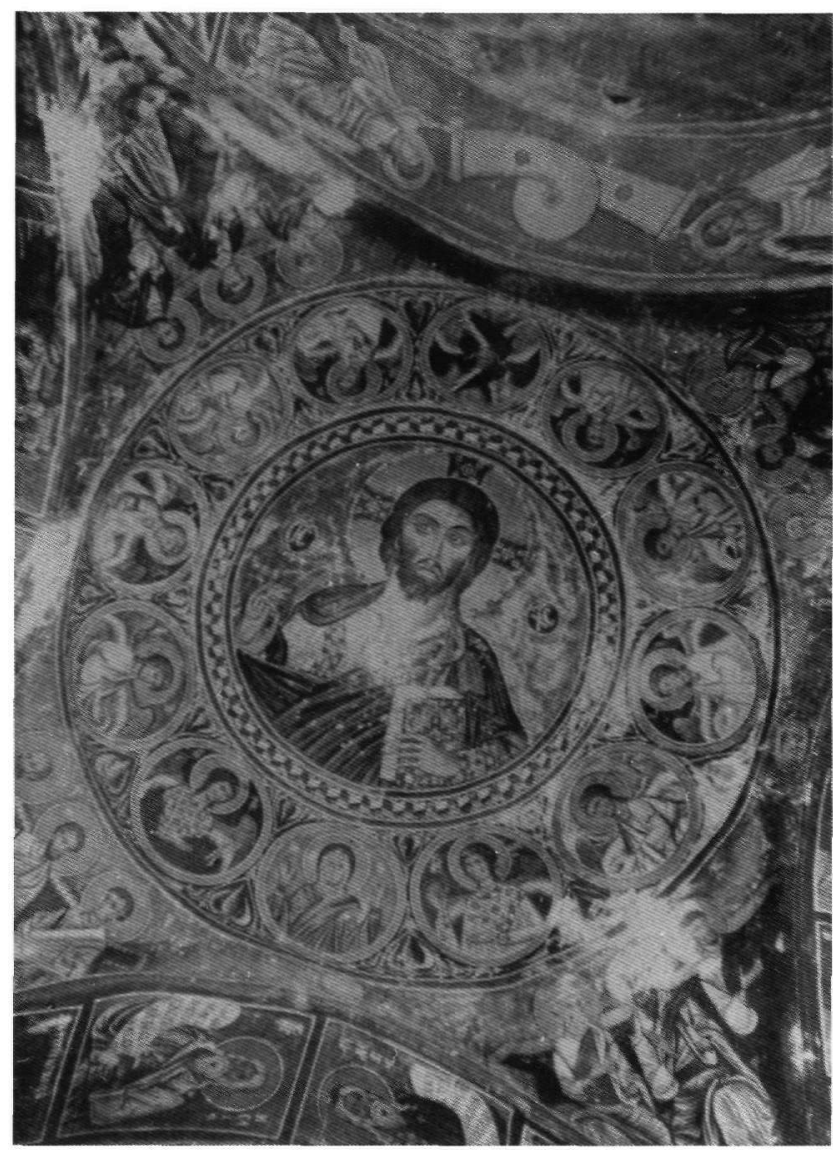

Fig. 13. Eglise de la Vierge d'Asinou, Chypre (narthex). Anges enroulant le ciel.

partie de l'église, des sujets se rapportant au dogme, traités en compositions symétriques. Sa familiarité avec les conceptions de l'époque se reflète également dans la part d'innovation dont témoignent autant la figuration des signes célestes - qui, suivant en cela la tendance générale de l'art paléologue, figurent en personnifications ailées - que, et surtout, la forme du disque qui les contient: celui-ci a l'aspect d'une larme (ce que l'on distingue assez mal sur le dessin) ${ }^{23}$, allusion (comme l'a suggéré Sr. Djurić) à leur caractère astronomique, explicable justement par l'intérêt des intellectuels de l'époque - tels Jean Pediasime, Maxime Planudès, Manuel Bryennios ou Théodore Methochite - pour l'astrologie $^{24}$. Cette image savante de Bogorodiča Ljeviška, sans avoir été reprise dans toute sa complexité, a inspiré d'autres monuments serbes importants. On la retrouve ainsi, en tableau autonome - non plus en surface incurvée mais sur le mur vertical ouest du narthex - à Gračanica (1320), accompagnée de l'inscription: anges du Seigneur (Fig. 11) ${ }^{25}$. Un peu plus tard, une composition du firmament qui se veut moins schématique, com- portant des nuages ainsi que les personnifications de la lune et du soleil, est incluse dans la représentation du Jugement Dernier à Dečani (1338-1347/8) ${ }^{26}$ (Fig. 12). Par la disposition formelle de ses éléments iconographiques - anges encadrant le firmament et l'enroulant - cette intéressante image n'est pas sans rappeler celle de Müstair (Fig. 4), même si, dans le monument serbe, on insiste sur l'aspect "détaché» du ciel.

Cette même variante, plus structurée que la composition de Saint-Nicolas tis Stégis, accompagnée de l'inscrip-

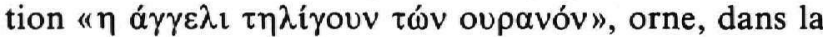
même île, l'intrados de l'arc au-dessus de la lunette ouest du narthex de l'église de la Vierge d'Asinou $(1332 / 3)$ (Fig. 13) ${ }^{27}$, et se retrouve dans des manuscrits, comme l'Evangile 212 de Matenadaran à Erevan $(1337)^{28}$. Dans ces figurations, le rouleau du firmament forme une boucle, effet décoratif certes, mais qui montre surtout que le ciel est déjà défait et la fin du monde commencée, ce que l'on voit déjà esquissé en images, deux siècles plus tôt, à Saint-Nicolas de Kakopetria (Fig. 8). Remarquons que, dans l'inscription qui accompagne la scène dans cette dernière église, dans celle d'Axtala ainsi que dans l'église de Hagios Stephanos à Kastoria dont on parlera plus loin, le texte apocalyptique est plus ou moins respecté, alors que sur les décors du XIVe siècle (Gračanica, Asinou, SaintGeorges de Babylas) est soulignée la présence des anges ainsi que, dans les deux derniers exemples cités, leur fonction. Mentionnons enfin, à titre indicatif, un dernier exemple byzantin, connu et daté, de cette même variation, et qui se trouve en Crète, dans l'église de SaintGeorges à Artos (région de Réthymnon) ${ }^{29}$. Reproduisant, comme à Gračanica, des anges en plein vol sur une surface verticale, cette composition couronne la Déisis du mur ouest du narthex.

Pour revenir à l'image de l'église de Saint-Georges (Fig. 1) qui a servi de point de départ à cette étude, les deux anges - cas unique, semble-t-il, pour la région ${ }^{30}-s^{\prime} y$ partagent la surface disponible de la scène, enroulant, chacun de son côté, la bande du firmament. Ils figurent en contraposto et se penchent - chacun à un degré différent - vers le centre de la composition: leur grande enjambée ne s'arrête que parce que leurs pieds, avancés jusqu'à l'axe vertical de la composition, se touchent et que leur tête inclinée a atteint l'axe vertical de leur point d'appui. Chacun des deux corps décrit ainsi un arc, et tous deux entourent en un cercle le rouleau du ciel, centre noétique de la composition, réhaussé également par sa couleur lumineuse. Cette courbure des corps presque symétriques, soulignée par la bordure de manteaux, est mise encore en valeur par les plis des chitons, plus lourds pour l'ange de droite, plus souples pour 


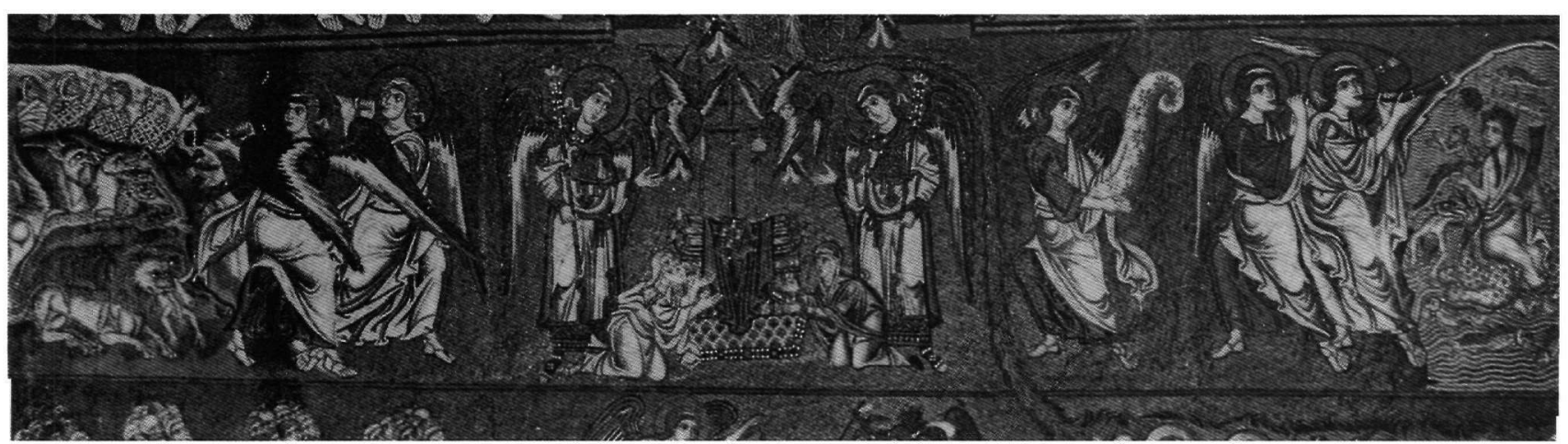

Fig. 14. Torcello. Jugement Dernier (détail).

celui de gauche. On pourrait noter ici que ce mouvement vif qu'ils viennent d'achever a, en même temps, quelque chose de figé, ce qui achève de conférer à ces anges l'impression de figures arrêtées dans le temps. Quant au rouleau firmament, entouré autour de l'omphalos que l'on voit dans les mains de l'ange de gauche, il est sur le point d'être presque complètement enroulé, seul un morceau de ciel étant encore visible où figure un soleil au profil presque caricatural. Ainsi s'amorce la fin des temps, et la détresse cosmique annoncée par les textes.

Pour composer l'image de l'église de Saint-Georges de Babylas, le peintre, bien qu'ayant emprunté à des anges debout et en mouvement la composition de la partie basse du corps de ses figures - comme le montre la comparaison avec, entre autres, le manuscrit de la Walters Art Gallery à Baltimore W 539 (Fig. 9) - a abouti à une composition dont le caractère quasiment statique est renforcé par la symétrie des postures de ces anges. Par leur tête fortement inclinée, touchant presque leurs bras soulevés pour effectuer l'enroulement, ces figures donnent l'impression de reproduire, du point de vue formel, un des types iconographiques exprimant la tristesse dans le répertoire des gestes de l'art byzantin établi par Henry Maguire. On pourrait ainsi rapprocher la représentation des pénitents du Vat. gr. 1754 de l'impression que donne la position de nos anges, laquelle transmettrait ainsi, presque malgré elle, ce sentiment de tristesse retenue ${ }^{31}$.

23. Sr. Djurić, The Representations of Sun and Moon at Dečani, Dečani et l'art byzantin au milieu du XIVe siècle (Dečani 1985), Beograd 1989, p. 342-343, n. 38, fig. 4-7.

24. Ibid., p. 343, n. 43 à 46. Cf. également I. N. Pontikos, 'Avwvú-

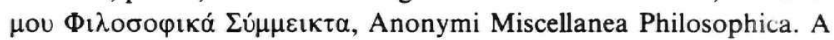
Miscellany in the Tradition of Michael Psellos (Codex Baroccianus graecus 131), Paris-Bruxelles 1992, p. XLIII.

25. Br. Zivković, Gračanica. Les dessins des fresques. Les monuments de la peinture serbe médiévale, 7, Beograd 1989, X, Narthex. Je dois cette traduction au R. P. Christopher Walter que je remercie également pour ses conseils. Je remercie aussi M. Titos Papamastorakis d'avoir attiré mon attention sur cette image. Cette inscription, qui accompagne souvent les anges (O. Demus, The Mosaics of Norman Sicily, London 1949, p. 39, pl. 14) est moins appropriée que celles qui, décrivant la scène, se réfèrent spécifiquement à leur fonction; cf. note 27 .

26. Djurić, op.cit., p. 343 , fig. 8.

27. W. H. Buckler, The Church of Asinou, Cyprus, and its Frescoes. Archaeologia 83 (1933), p. 339 et pl. XCIII.2. Les anges sur l'intrados de l'arc ouest font pendant aux deux prophètes à rouleaux déployés qui occupent la place opposée de l'arc est. Cf. également l'image dans H. Maguire, Art and Eloquence in Byzantium, Princeton 1981, fig. 45. Ce verbe $\tau u \lambda i \sigma \sigma \omega$, autre forme du verbe du texte de l'Apocalypse ( $\varepsilon i \lambda i \sigma \sigma \omega)$, est attesté au XIIe siècle: H. Stéphanus, Thesaurus Graecae Linguae, VII, s.v.

28. Velmans, L'image de la Déisis, op.cit. (note 7), p. 151, fig. 33. 29. Les anges, symmétriquement disposés, comme dans un décor crétois plus ancien, au-dessus de la fenêtre, s'harmonisent, encore ici,

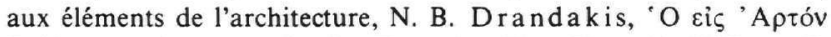

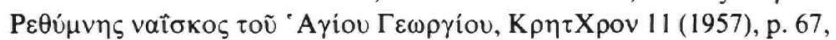
145 pl. 14.1. Ch. Ranoutsaki, Die Fresken der Soteras ChristosKirche bei Potamies, MiscByzMon 36, 1992, p. 112, fig. 31 (fin XIVe s.). Je remercie Mme C. Mylopotamitaki d'avoir attiré mon attention sur cette étude.

30. Dans les monuments connus du Péloponnèse, on ne rencontre, en effet, que la figuration plus simple à ange unique (comme, entre autres, à Saint-Stratège de Boularii dans le Magne - couche du XIIe siècle), et celà même dans le cas d'églises importantes, comme la Mé-

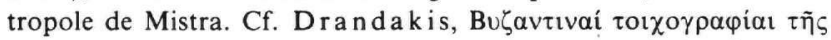

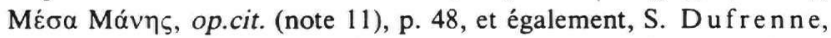
Les programmes iconographiques des églises byzantines de Mistra, Paris 1970, schéma VIb, n. 20.

31. $F^{\circ} 6 \mathrm{v}$; surtout les première et troisième figures de gauche qui appuient leur tête sur leur main. J. R. Martin, The Illustration of the Heavenly Ladder of John Climacus, Princeton 1954, 134, fig. 256. H. Maguire, The Depiction of Sorrow in Middle Byzantine Art, DOP 31 (1977), p. 133 n. 48 et fig. 55. Toujours sur le plan formel, c'est par le même geste, par exemple, que le Juif qui enlève la plaque du tombeau de Lazare exprime la tristesse sur l'icône du Sinaï du XIIe siècle: K. Weitzmann, M. Chatzidakis, K. Miatevet S. Radojčić, Les icônes, Belgrade 1966, fig. 25. Il persiste évidement le problème de surinterprétation d'images éloignées chronologiquement de leurs sources écrites. Sur la rhétorique des images voir le compte rendu du livre de H. Maguire (note 27) dans GOthR 30. 1 (1985), p. 89-90. Pour la période voir G. Hellemo, Adventus Domini. Eschatological Thought in 4th-Century Apses and Catecheses, Köln 1989, p. 146-158. 
Originellement conçue pour des surfaces incurvées, mais transposée ici sur une surface verticale, cette composition ne peut plus bénéficier des possibilités offertes par les surfaces courbes ${ }^{32}$; elle semble réussir, en revanche, à introduire un nouveau paramètre: ce sentiment de tristesse de Fin des Temps qui parcourt les textes et qu'on ne retrouve pas sur la composition analogue à Gračanica figurée également sur une surface verticale (Fig. 11). Du reste, contemporain d'Ephrem le Syrien - sous le nom duquel s'est constitué le premier corpus de textes qui ont inspiré le Jugement Dernier - Cyrille de Jérusalem, se réfère, lui aussi, à cette même image eschatologique d'Isaïe 34, 4, puis de Matthieu 24, 29, qu'il commente - imité en cela par d'autres auteurs ${ }^{33}$ — en un texte qui a servi d'en-tête à notre article. Ainsi, la fonction de la variante de notre église serait peut-être double: rôle formel et rôle sémantique.

Il semble ainsi que, dès lors qu'il s'agit d'examiner les origines de cette scène inspirée par l'Apocalypse 6, 4, il faille - au-delà de la question soulevée par Sehoko Tsuji ${ }^{34}$ : un ou deux anges? - tenir compte également de leurs différentes postures. Deux types pourraient ainsi être dégagés:

- Premièrement, les anges debout et statiques encadrant le rouleau du ciel, comme dans leur représentation la plus ancienne (Müstair), type que l'on entrevoit à Chypre au XIIe siècle (Fig. 8), puis, deux siècles plus tard, à Dečani (Fig. 12); peut-être pourrait-on le mettre également en relation avec le schéma correspondant de Kokkinobaphos.

- Ensuite, l'ange unique et en mouvement des premiers exemples du XIe siècle, soit qu'il entraîne derrière lui ou vers le haut le rouleau du ciel "défait", soit qu'il se retourne pour effectuer le même mouvement (Fig. 2-3). Les origines formelles de cette variante, moins proche, on l'a vu, de l'idée d'enroulement du firmament, sont encore incertaines.

Dans son article, Herbert Hunger - afin d'expliquer la présence des anges dans l'iconographie de la scène alors que ceux-ci ne sont pas mentionnés dans les textes propose, comme source d'inspiration, les anges appelant les élus ${ }^{35}$. Ce thème, auquel se réfère Ephrem et qui se trouve dans l'Apocalypse $(8,2-6)$ mais également dans les rextes évangéliques (Mtth. 24, 31) et pauliniens (A Cor. 15, 52; A Thess. 4, 16), est traduit en image par l'ange buccinateur et fait aussi partie de la tradition iconographique de la Seconde Venue. Comme on le sait, ce motif iconographique trouve assez tôt sa place dans le cadre du Jugement Dernier, dès l'époque paléochrétienne $^{36}$, et se rattache également à la Résurrection des Morts de la Terre ou de la Mer. En règle générale, est figuré un ange (ou, plus rarement, deux), qui, très sou-

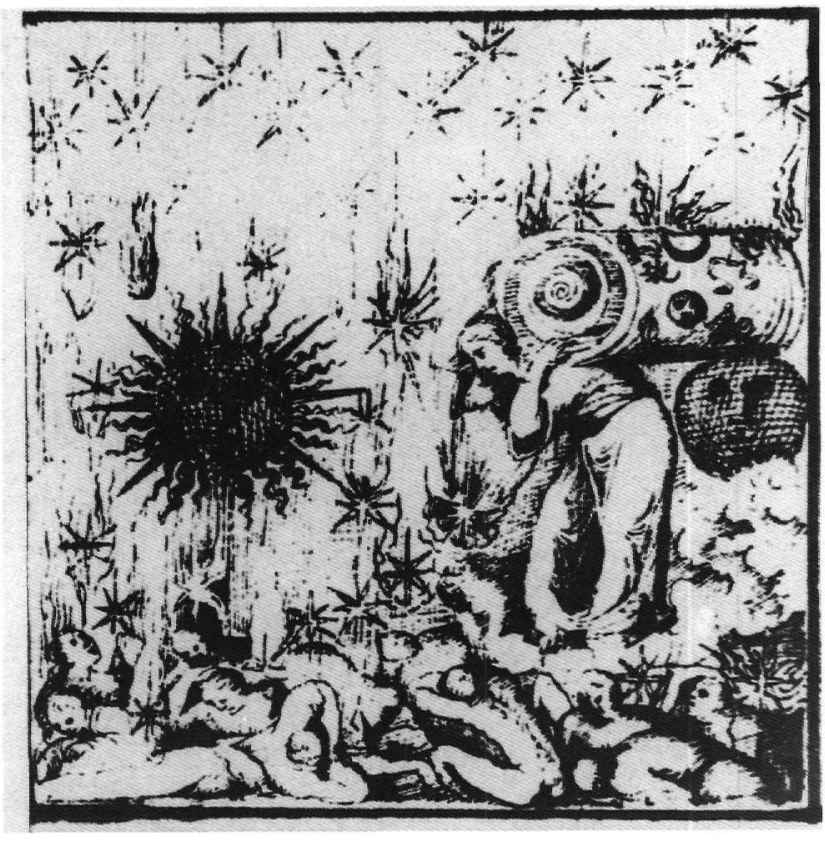

Fig. 15. Venise, Marc. VII, $22(=1466), f^{\circ} 195 r$. Ange portant le rouleau du ciel.

vent, se retourne pour souffler dans la trompette, dans un mouvement imprégné, presque toujours, d'un certain dynamisme. On remarque également que, dans ces deux motifs - anges soufflant de la trompette et anges enroulant le ciel — presque toujours jumelés dans le Jugement Dernier, les anges partagent, dès le XIe siècle, les mêmes postures et gestes, comme le montrent les exemples de Saint-Demètre à Vladimir et de Torcello (Fig. 14) ${ }^{37}$. Si l'hypothèse de Herbert Hunger - emprunt des seconds aux premiers - est correcte, il est aisé de comprendre pourquoi, dans les premiers exemples du XIe siècle (Fig. 2-3) le rouleau de ciel que tient l'ange, imité de celui qui sonne de la trompette - acte en soi triomphant - donne moins l'idée de l'enroulement du ciel que celle,

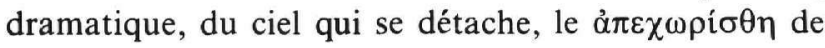
l'Apoc. 6, 14.

Plus près du texte de l'Apocalypse semble l'image des anges de ce que l'on a mentionné comme "premier type" ", c'est-à-dire deux anges statiques qui enroulent le ciel. Peut-on attribuer à cette variation formelle une origine distincte et suggérer un emprunt, non plus au cycle du Jugement Dernier (ange soufflant de la trompette: hypothèse plausible de Hunger), mais en dehors de celui-ci? Pour revenir de nouveau aux images de Müstair et de Kokkinobaphos, peut-on relever entre elles des analogies formelles, même si dans le dernier manuscrit les anges n'enroulent pas le firmament ${ }^{38}$ mais le soulèvent, et même si cette composition, dépassant de deux siècles le modèle supposé de la fresque de Müstair, 
s'éloigne très sensiblement de la représentation figurant habituellement dans le Jugement Dernier? En effet, bien que ces deux schémas ne soient pas identiques, ils reproduisent tous deux un élément iconographique important. Il s'agit du firmament constellé qui, dans le manuscrit, figure sous le forme de $\pi \alpha \rho \alpha \pi \varepsilon \dot{\tau} \alpha \sigma \mu \alpha$ (reprenant ainsi la symbolique chrétienne ${ }^{39}$ ), et dont l'existence serait probablement à expliquer par une source iconographique commune, provenant de l'illustration scientifique, c'est-à-dire la Création de l'Univers dans les Octateuques byzantins (Fig. 6). Toujours est-il que, même si la source est commune, l'image du firmament se matérialise chaque fois différemment, le peintre carolingien s'étant efforcé, par exemple, de reproduire un rouleau au sens strict du terme, suivant en cela le sens littéral du texte à illustrer, alors que l'image de Bačkovo reproduit l'aspect d'une longue écharpe flottant vers le haut que l'ange se hâte de saisir. De même, la disposition symétrique des luminaires sur l'étoffe tendue du Par. gr. 1208, et qui rappelle également l'imagerie des Octateuques, diffère également des rouleaux du ciel analogues du Jugement Dernier. En effet, dans ces derniers, soit les luminaires sont absents du rouleau du ciel comme à Müstair ou Kakopetria - soit le rouleau figure en volumen peu large, déroulé, mais d'un aspect fort différent, et où le soleil et la lune tiennent une place peu importante et comme fortuite. Enfin, si, dans les divers Jugements Derniers, la couleur bleue du ciel reproduite dans le Par. gr. 1208 et le Vat. gr. 747 n'est pas franchement reprise, cette différence pourrait très bien ne refléter que le désir d'imiter la couleur du rouleau en parchemin.

Si l'on considère que le rouleau de Müstair demeure un cas unique jusqu'au XIe siècle (ce motif est absent, par exemple, des Jugements Derniers de Güllü Dere - 913/ 920 - et de Yilanli Kilisé - 2e moitié du Xe s.), cette scène des anges enroulant le ciel ne semble faire véritablement son apparition dans les représentations connues - peu nombreuses, certes - du Jugement Dernier qu'à cette époque, peut-être sous l'influence du renouveau de l'astrologie à cette même période ${ }^{40}$. C'est ainsi que, lorsqu'au Xe siècle, le peintre de Hagios Stephanos de Kastoria peint la scène, l'enroulement du ciel est plus suggéré par l'inscription qui parcourt le segment de fir-

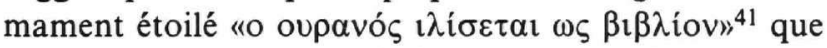
par le geste à peine esquissé des anges statiques. Deux siècles plus tôt déjà, lorsque le moine Jean de Jérusalem ${ }^{42}$ décrit une représentation du Jugement Dernier, la scène des anges enroulant le ciel n'est pas incluse. Seraitce qu'à la réticence habituelle des peintres à illustrer l'Apocalypse se serait ajoutée, pour ce verset, la difficulté de le traduire en images, ce qui expliquerait sa rareté
32. O. Demus, Byzantine Mosaic Decoration, London 1953, p. 9 , décrivait déjà les conséquences esthétiques du passage de configurations conçues pour les surfaces incurvées à des surfaces plates.

33. 15ème Catéchèse Baptismale III, 4-5, PG 33, 873, A-B (où il se réfère aux textes d'Isaïe et de Matthieu). Hung e r, op.cit. (note 3), p. 80 n. 3. Plus tard, André de Césarée fera le même commentaire, cf. N. Thierry, L'Apocalypse de Jean et l'iconographie byzantine, dans: L'Apocalypse de Jean. Traditions exégétiques et iconographiques, IIIe-XIIIe siècles (Actes du Colloque de la fondation Hardt, 29 février-3 mars 1976), Genève 1979, p. 325 n. 14.

34. Tsuji, op.cit. (note 7), p. 73, n. 6.

35. Hunge r, op.cit., p. 81, n. 5. Dans ce texte d'Ephrem le Syrien, la trompette est mentionnée de façon générale, suggérant le rôle des

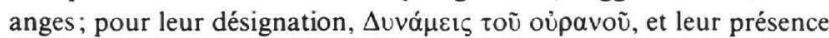
dans le Jugement Dernier, cf. D. Pall as, Himmelsmächte, Erzengel und Engel, RbK III, 1978, p. 17, 19.

36. Le motif existe déjà au VIe siècle, avec des anges qui illustrent Apoc. 8, 2-6, dans l'église de Saint-Michel in Affricisco à Ravenne. Pour de nouveaux apports sur l'iconographie et la datation de ces mosaïques, Y. Christe, Nouvelle interprétation des mosaïques de Saint-Michel in Affricisco à Ravenne : à propos d'Ap. VIII, 2-6, RAC LI (1975), fig. 1, 109 n. 4 et 122, où l'auteur suppose une éventuelle réfection au IXe siècle et détache cette représentation de l'iconographie de la Parousie; au VIIe siècle sont également représentés des anges buccinateurs - qui, ici, se détournent - sur le sarcophage de Jouarre, dans le cadre du Jugement Dernier. Cf. B renk, Tradition und Neuerung (note 7), p. 43-44, pl. 16 et 6 respectivement. Pour un bon dessin de l'ange, cf. ide m, Marginalien zum sog. Sarkophag des Agilbert in Jouarre, CahArch 14 (1964), p. 100, fig. 2.

37. V. Lazarev, Old Murals and Mosaics, London 1966, fig. 65, 67. I. Andreescu, Torcello. I. Le Christ inconnu. II. Anastasis et Jugement Dernier. Têtes Vraies, Têtes Fausses, DOP 26 (1972), fig. 15.

38. Suggéré par Djurić, op.cit. (note 23), p. 342.

39. Selon celle-ci, le $\pi \alpha \rho \alpha \pi \dot{\varepsilon} \tau \sigma \mu \alpha$ (ou, plus souvent, $\kappa \alpha \tau \alpha \varepsilon \dot{\varepsilon} \tau \alpha \sigma \mu \alpha$ ), sépare la terre de la voûte céleste. Pour ces deux termes, cf. G. W. H. L a m pe, A Patristic Greek Lexicon, Oxford 1956, s.v. D'après Hu tter, op.cit., p. 350 n. 4, qui s'interroge sur un éventuel apport iconographique du rouleau du ciel du Jugement Dernier, que finalement elle ne retient pas, la présence de ce rideau du firmament reflèterait des apports iconographiques de l'imagerie impériale (ibid., p. 348-352). 40. Pour le sujet, voir l'article récent de Pontik os, op.cit. (note 24), p. XLII, XLIII. Pour l'importance de l'astrologie au siècle suivant,

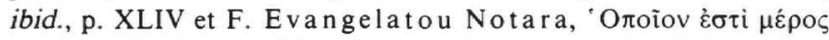

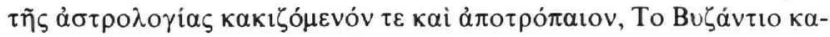

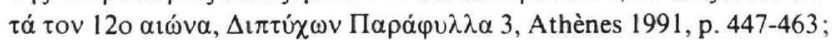
cet interêt transparaît jusque dans la décoration des églises, comme sur le pavement du narthex de Pantocrator à Constantinople (monastère où vivait le moine auteur de la lettre à Manuel I fustigeant l'astrologie et mentionnée par Evangelatou Notara, op.cit., p. 451, n. 20); A. H. S. Megaw, Notes on Recent Work of the Byzantine Institute in Istanbul, DOP 17 (1963), p. 337, fig. A; A. P. Kazhdan et Ann Wharton Epstein, Change in Byzantine Culture in the Eleventh and Twelfth Centuries, Londres 1985, p. 150, fig. 14.

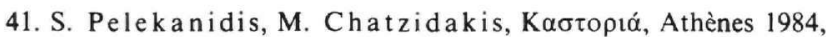
p. 7, 18 fig. 6, et M. Panayotidi, La peinture monumentale en Grèce de la fin de l'Iconoclasme jusqu'à l'avènement des Comnènes (843-1081), CahArch 34 (1986), p. 79 n. 20, et p. 80, 82. Ces auteurs proposent la seconde moitié du Xe siècle; on ne peut, pour le moment, se prononcer sur la date 889 avancée avec précaution par N. K.

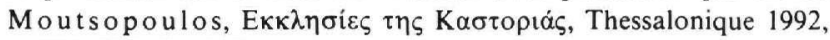
p. 298,299 , fig. 248 .

42. Auteur présumé d'une oraison qui serait datée de 764. Cf. H u n- 
jusqu'au XIe siècle ainsi que son absence dans certains monuments plus tardifs ${ }^{43}$ ?

Il apparaît ainsi, d'une part, que ces divers efforts d'illustration de ce texte apocalyptique - qui, selon Brenk, aurait déjà été traduit en images vers la seconde moitié du VIIIe siècle - se seraient inspirés de deux sources iconographiques: a) d'une part, l'ange sonnant de la trompette qui, traditionnellement, fait partie du Jugement Dernier et dont la transformation en ange à rouleau du ciel a pu être facilitée par la figuration des rouleaux des prophètes, hypothèse qui corroborerait celle de Herbert Hunger; b) d'autre part, des images qui, comme dans le Kokkinobaphos (et avant lui, au XIe siècle, l'Octateuque Vat. gr. 747) transmettraient la tradition hellénistique - optique ou scientifique, quoique simplifiée — du firmament étoilée que reproduit le rouleau du ciel du Jugement Dernier.

Par ailleurs, tous les exemples connus du schéma de deux anges étant situés aussi bien dans les Balkans et à Chypre que dans la périphérie (Géorgie ou Arménie), il semble qu'il faille supposer une source d'inspiration commune qui se situerait à Constantinople. Certes, dans la capitale même, la seule composition correspondante, celle de la Kariye Djami (1315-1321) - mais que l'on rencontre déjà au XIIIe siècle, comme à Sainte-Sophie de Trébizonde, réplique probable d'une église constantinopolitaine de la même époque ${ }^{44}-$ semble, par son ciel complètement enroulé et porté par un seul ange, s'inscrire en faux contre cette hypothèse, d'autant plus que le soleil et la lune qui y figurent ne reflètent en rien l'aspect astronomique du firmament caractéristique de cet engouement croissant des intellectuels pour l'astrologie au XIVe siècle. Cependant, cette apparente contradiction peut aisément s'expliquer. D'une part, la tradition choisie par le peintre de la Kariye Djami lui permet beaucoup mieux que le nouveau schéma d'intégrer la scène dans l'espace circulaire à décorer. D'autre part, cette composition, pour originale qu'elle paraisse, ne représente vraisemblablement qu'une simple évolution de la tradition antérieure (rouleau porté par un ange) que l'on retrouve même à l'époque post-byzantine ${ }^{45}$ (Fig. 15) - parallèlement au nouveau schéma icono- graphique en émergence. Rien n'exclut donc que l'on puisse situer la variante typologique de deux anges enroulant le ciel dans la tradition de Constantinople, centre fort probable de la cristallisation et de la diffusion de la composition du Jugement Dernier.

ger, op.cit. (note 3), p. 82 n. 2.

43. Hunger, op.cit., p. 81. Pour des monuments où le sujet iconographique n'est pas reproduit, cf. note 10. Thie r ry, op.cit. (note 33), p. 320 et 325 .

44. Underwood, op.cit. (note 6), p. 201; 3, (204)-I. Ce genre de figuration du ciel n'est pas sans rappeler l'aspect imagé du oủpóvıov

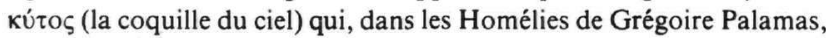
se rétrécit à l'approche de l'Antichrist, PG 151,152. Hunge r, op.cit., p. 81. Sur la voûte du porche ouest de l'église de Trébizonde - monument qui s'inspire de l'art de la capitale - l'emplacement de la composition a inspiré également la disposition analogue du chœur des élus: cf. D. Talbot Rice, The Church of Haghia Sophia at Trebizond, Edinburgh 1968, p. 148 n. 30, fig. 110.

45. Cela rappelle, par ailleurs, le ciel sous forme de disque, que l'on rencontre antérieurement - encore que toujours dans un contexte complètement différent - comme, par exemple, à la Chapelle Palatine (O. Demus, The Mosaics of Norman Sicily, London 1949, p. 254, pl. 26B), mais aussi à Constantinople même (cf. note 40). L'utilisation du schéma à un ange pouvait également être facilement adaptable pour la figuration d'un ciel complètement enroulé qui, par son emplacement au centre de la scène, revêt ainsi - surtout à Kariye Djami - tout son sens théologique et sotériologique. Cf. RAC XV (1991), p. 188 et 197 . Cette figuration à ange unique se retrouve chez le peintre crétois Georges Clontzas, qui, influencé parfois par l'iconographie occidentale, inclut ce motif dans ses différentes compositions du Jugement Dernier - sujet qui lui est cher - s'inspirant du schéma

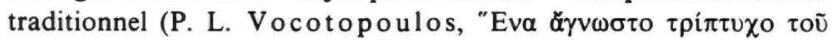

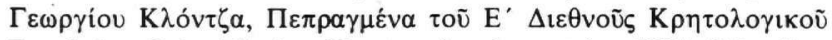
$\Sigma \cup v \varepsilon \delta$ píou (Saint Nicolas, 25 septembre-ler octobre 1981), Héraclion 1985 , B', p. 69 , fig. 5), tout en créant parfois ses propres compositions - tel l'ange sans ailes portant sur son dos le rouleau du ciel rendu en perspective sur le codex VII, $22(=1466)$ de la Marcienne de Venise, $\mathrm{f}^{\circ}$

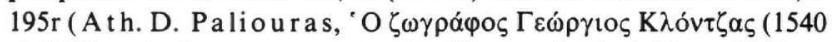

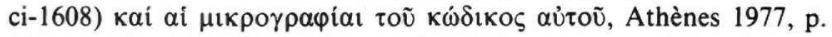
184, n. 7, fig. 393). Enfin, l'illustration de l'ange sur une icône de Symi (ange également sans aile, ciel en forme de globe), attribuée au même peintre est si éloignée des schémas traditionnels que la piété populaire pourra y voir tout autre chose (cf. M. A cheimastou, Eik $\dot{\omega} v \tau \tilde{\eta} \zeta$

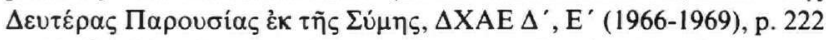
n. 45 , fig. $88 \alpha$ ). Pour la place du Jugement Dernier dans l'œuvre du peintre, cf. M. Chat zida k is, Icônes de Saint-Georges de Grecs et de la Collection de l'Institut, Venise 1962, p. XXXIII-XXXV. 